\title{
Chemotherapy sensitization of glioblastoma by focused ultrasound-mediated delivery of therapeutic liposomes
}

\section{Journal Article}

\section{Author(s):}

Papachristodoulou, Alexandros; Signorell, Rea D.; Werner, Beat; Brambilla, Davide; Luciani, Paola; Çavuşoğlu, Mustafa; Grandjean, Joanes; Silginer, Manuela; Rudin, Markus; Martin, Ernst; Weller, Michael; Roth, Patrick; Leroux, Jean-Christophe (D)

Publication date:

2019-02-10

Permanent link:

https://doi.org/10.3929/ethz-b-000315049

\section{Rights / license:}

Creative Commons Attribution-NonCommercial-NoDerivatives 4.0 International

\section{Originally published in:}

Journal of Controlled Release 295, https://doi.org/10.1016/j.jconrel.2018.12.009

\section{Funding acknowledgement:}

147651 - Focused Ultrasound-Mediated Delivery of Encapsulated MGMT Antagonists for the Treatment of TemozolomideResistant Glioblastoma (SNF) 

delivery of therapeutic liposomes

3 Alexandros Papachristodoulou ${ }^{\mathrm{a}, 1 \#}$, Rea Deborah Signorell ${ }^{\mathrm{b} \#}$, Beat Werner ${ }^{\mathrm{c}}$, Davide

4 Brambilla ${ }^{b, 2}$, Paola Luciani $i^{b, 3}$, Mustafa Cavusogluc ${ }^{c}$, Joanes Grandjean ${ }^{\mathrm{d}, 4}$, Manuela

5 Silginera, Markus Rudin ${ }^{\mathrm{d}}$, Ernst Martin ${ }^{\mathrm{c}}$, Michael Weller ${ }^{\mathrm{a}}$, Patrick Roth ${ }^{\mathrm{a}^{*}}$ and Jean-

6 Christophe Leroux ${ }^{\mathrm{b}+}$

8 aLaboratory for Molecular Neuro-Oncology, Department of Neurology, University Hospital

9 and University of Zurich, Frauenklinikstrasse 26, 8091 Zurich, Switzerland

bInstitute of Pharmaceutical Sciences, Department of Chemistry and Applied Biosciences,

ETH Zurich, Vladimir-Prelog-Weg 1-5, 8093 Zurich, Switzerland

${ }^{\circ}$ Center for MR-Research, University Children's Hospital Zurich, Steinwiesstrasse 75, 8032

13

14

Zurich, Switzerland

dInstitute for Biomedical Engineering, University and ETH Zurich, Gloriastrasse 35, 8032

Zurich, Switzerland

${ }^{*}$ Corresponding author: Email address: patrick.roth@usz.ch

Authorship notes: ${ }^{A}$ A. Papachristodoulou and R.D. Signorell are co-first authors. ${ }^{+}$. . Roth and J.-C. Leroux are co-senior authors

Keywords: Glioblastoma, temozolomide resistance, methylguanine-DNA

methyltransferase inactivation, liposomes, focused ultrasound, image-guided targeting ${ }^{1}$ Present address: Departments of Urology and Pathology and Cell Biology, Herbert Irving Comprehensive Cancer Center, Columbia University, 1139 St. Nicholas Avenue, 10032 New York, USA

${ }^{2}$ Present address: Faculty of Pharmacy, University of Montreal, H3T 1J4 Montreal, Canada ${ }^{3}$ Present address: Institute of Pharmacy, Department of Pharmaceutical Technology, Friedrich Schiller University Jena, 07743 Jena, Germany

${ }^{4}$ Present address: Singapore Biolmaging Consortium, Agency for Science Technology and Research, 138667 Singapore, Singapore 


\section{1}

2

8 inactivator in mice bearing temozolomide-resistant gliomas. We demonstrate that a

9 liposomal $\mathrm{O}^{6}$-(4-bromothenyl)guanine $\left(\mathrm{O}^{6} \mathrm{BTG}\right)$ derivative can efficiently target MGMT,

\section{Abstract}

In glioblastoma, the benefit from temozolomide chemotherapy is largely limited to a subgroup of patients (30-35\%) with tumors exhibiting methylation of the promoter region of the $\mathrm{O}^{6}$-methylguanine-DNA methyltransferase (MGMT) gene. In order to allow more patients to benefit from this treatment, we explored magnetic resonance image-guided microbubble-enhanced low-intensity pulsed focused ultrasound (LIFU) to transiently open the blood-brain barrier and deliver a first-in-class liposome-loaded small molecule MGMT thereby sensitizing murine and human glioma cells to temozolomide in vitro. Furthermore, we report that image-guided LIFU mediates the delivery of the stable liposomal MGMT inactivator in the tumor region resulting in potent MGMT depletion in vivo. Treatment with this new liposomal MGMT inactivator facilitated by LIFU-mediated blood-brain barrier opening reduced tumor growth and significantly prolonged survival of glioma-bearing mice, when combined with temozolomide chemotherapy. Exploring this novel combined approach in the clinic to treat glioblastoma patients with MGMT promoter-unmethylated tumors is warranted. 9

0

1

\section{3} 4 25 (1) 
2 Glioblastoma is the most aggressive type of glial neoplasm and therefore classified as a

\section{Introduction} World Health Organization (WHO) grade IV tumor. It represents the majority of all gliomas in adults, being the most frequent primary grade IV tumor of the brain [1]. After establishing the diagnosis by surgical resection or biopsy, the current treatment strategy for newly diagnosed glioblastoma patients aged 70 or younger includes radiotherapy and temozolomide (TMZ) alkylating chemotherapy [2].

Although the current standard of care for patients with newly diagnosed glioblastoma has improved with the addition of $\mathrm{TMZ}$ [3], the overall survival benefit remains moderate and mainly restricted to a subpopulation of patients with MGMT promoter-methylated tumors [4]. Due to its clinical relevance, present clinical trials are stratifying patient populations according to their MGMT promoter methylation status [5]. Most glioblastoma patients harbor a tumor with an unmethylated MGMT promoter leading to MGMT gene expression [6] and subsequent ability to repair the $\mathrm{O}^{6}$-methylguanine $\left(\mathrm{O}^{6}-\mathrm{meG}\right)$ cytotoxic lesions caused by TMZ. Since no other pharmacological agent has been demonstrated to prolong overall survival in glioblastoma patients, a major research focus has been to overcome MGMT-mediated TMZ resistance. This can be achieved by targeting MGMT with pseudosubstrates leading to its depletion. Agents like $\mathrm{O}^{6}$-benzylguanine act as substrate analogues to $\mathrm{O}^{6}-\mathrm{meG}$ lesions: they bind MGMT and subject it to ubiquitin-mediated degradation [6]. However, the clinical use of $\mathrm{O}^{6}$-benzylguanine yielded disappointing results mainly due to its dose-limiting systemic myelotoxicity when combined with TMZ [7]. Similar MGMT inactivators (e.g. $\mathrm{O}^{6} \mathrm{BTG}$ ), were developed for central nervous system and other solid tumors, in attempts to overcome this limitation but resulted in similar outcomes [8], precluding further clinical development.

The formulation of MGMT inactivators into liposomal nanocarriers can potentially improve 6 their pharmacokinetic properties, biodistribution profiles and decrease systemic dose- 
1 limiting adverse effects [9]. Liposomes are submicron-sized spherical nanocarriers

2 consisting of an aqueous core confined by a phospholipid bilayer that can protect the

3 encapsulated compound from plasma inactivation [10]. Poly(ethylene glycol) (PEG)-

4 coating of these nanocarriers reduces the clearance by the mononuclear phagocyte

5 system, thereby increasing their systemic circulation half-life [11]. Therapeutic agents

6 loaded in liposomes may represent a promising therapeutic strategy against glioblastoma,

7 especially if they are combined with methods mediating their delivery beyond the blood-

8 brain barrier (BBB) at the tumor site.

9 Albeit the BBB is not completely intact in the tumor region, macromolecular therapeutic

10 agents may still not reach gliomas in sufficient concentrations [9]. LIFU in combination with

11 systemically administered microbubbles can facilitate localized BBB opening under MR

12 imaging (MRI) guidance by exerting mechanical stress to the brain endothelium leading to reversible disruption of cellular tight junctions [10]. Importantly, feasibility studies have 


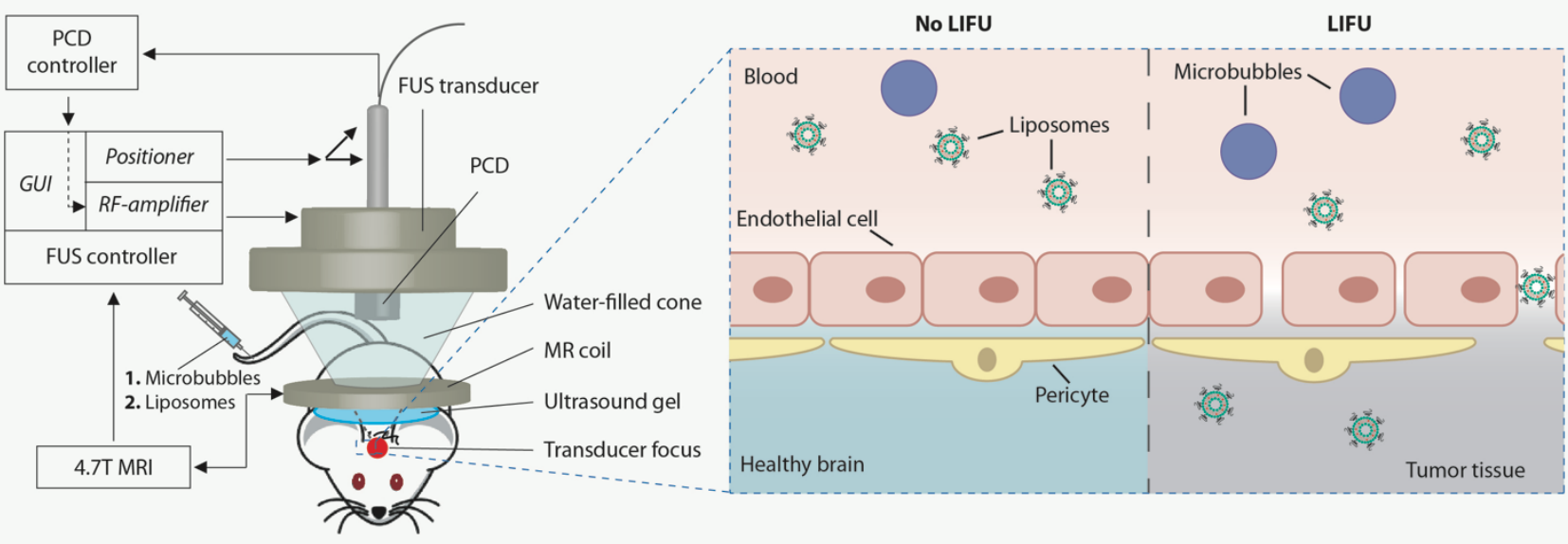

2 Figure 1. Schematic representation of MRI-guided LIFU-mediated liposome delivery across

the BBB. A dedicated rodent focused ultrasound (FUS) system integrated into a 4.7 T MR imaging system (MRI) allowed for graphical user interface (GUI)-controlled positioning of the FUS transducer and execution of low-intensity pulsed focused ultrasound (LIFU) sonications.

6 Microbubbles (1) and liposomal MGMT inactivator (2) were intravenously injected into

7 anaesthetized and stereotactically fixated (not shown) mice. Thirty seconds after onset of infusion,

8 the tumor region as identified on T2-weighted MRI was sonicated to induce BBB opening and

9 liposome influx to the tumor site. Safe sonication levels were ensured by driving the radio frequency (RF)-amplifier via an in-house developed passive cavitation detector ( $P C D)$ controller. 


\section{Materials and methods}

\subsection{Reagents and cell lines}

3 The mouse glioma cell line SMA-497 was derived from a spontaneous astrocytoma in a

$4 \quad$ VM/Dk mouse [18] and provided by Dr. D. Bigner (Duke University, Durham, NC). SMA497 cells were maintained in Dulbecco's modified Eagle's medium supplemented with $10 \%$ fetal calf serum (Biochrom, Minneapolis, MN) and 2 mM L-glutamine (Gibco Life Technologies, Paisley, UK). The glioma-initiating cell (GIC) line ZH-161 was established after informed consent and approval of the local ethic committees and previously characterized [19]. The cells were maintained in Neurobasal medium supplemented with 2 $\mu \mathrm{l} / \mathrm{ml}$ B-27 without vitamin A, 2 mM L-glutamine (Gibco Life Technologies), fibroblast growth factor (FGF)-2, epidermal growth factor (EGF) $(20 \mathrm{ng} / \mathrm{mL}$ each, Peprotech, Rocky Hill, PA). All cells were grown in a humidified $37^{\circ} \mathrm{C}$ incubator with $5 \% \mathrm{CO}_{2} . \mathrm{BG} 8235$ microbubbles were kindly provided by Bracco Suisse SA (Geneva, Switzerland) and show similar characteristics as Bracco's BR-38 microbubbles [20]. TMZ was kindly provided by Schering-Plough (Kenilworth, NJ) and prepared in stock solutions (100 mM) in dimethylsulfoxide (DMSO). Isolated human erythrocytes were supplied by Blutspende Zürich (Zurich, Switzerland).

\section{2. $\mathrm{O}^{6} \mathrm{BTG}-\mathrm{C} 18$ synthesis and liposome formulation}

${ }^{6}$ BTG-C18 was synthesized and loaded into liposomes to form LP-O'BTTG-C18 as described previously [17]. Briefly, O6 BTG (Cayman Chemical, Ann Arbor, MI) was incubated with 1-bromooctadecane (abcr GmbH, Karlsruhe, Germany) and lithium hydride (abcr $\mathrm{GmbH}$ ) in dimethylformamide for $3 \mathrm{~h}$ at $80^{\circ} \mathrm{C}$. After purification by silica gel column chromatography, $\mathrm{O}^{6} \mathrm{BTG}-\mathrm{C} 18$ was added at an initial molar drug-to-lipid ratio of $4 \mathrm{~mol} \%$ to a mixture of 1,2-dioleoyl-sn-glycero-3-phosphocholine (DOPC, Lipoid GmbH, Ludwigshafen am Rhein, Germany), cholesterol (Sigma Aldrich, St. Louis, MI) and 1, 2distearoyl-sn-glycero-3-phosphoethanolamine-poly(ethylene glycol 2000) (DSPE-PEG, 
1 Lipoid $\mathrm{GmbH}$ ) dissolved in chloroform at a molar ratio of 72.5:20:7.5. Liposomes were

2 formed after rehydration of the lipid film and immediately extruded to obtain vesicles with

3 sizes of $75 \mathrm{~nm} .1,1^{\prime}$-dioctadecyl-3,3,3',3'-tetramethylindodicarbocyanine, 4-

4 chlorobenzenesulfonate salt (ThermoFisher Scientific, Waltham, MA). (DiD)-labelled

5 liposomes were prepared as follows. Briefly, appropriate volumes of DOPC, CHOL, DSPE-

6 PEG, and DiD chloroform stock solutions were mixed to obtain a molar lipid ratio of

$7 \quad 52: 40: 7.5: 0.5$, respectively. After evaporating the organic solvent and complete drying

8 under vacuum overnight, the lipid film was rehydrated with HEPES-buffered saline (final

9 lipid concentration: 7.8 mM). Following seven freeze/thaw cycles, liposomes were

10 extruded 10 times with a Lipex $10 \mathrm{~mL}$ extruder (Lipex Biomembranes, Burnaby, BC,

11 Canada) through $0.05 \mu \mathrm{m}$ pore sized polycarbonate membranes (Sterlitech Corporation,

12 Kent, WA) and were stored at $4{ }^{\circ} \mathrm{C}$ until further use. The fluorescence intensity was

13 determined with an Infinite M200Pro plate reader (Tecan, Maennedorf, Switzerland) at $\lambda_{\mathrm{ex}}$

$14=600 \mathrm{~nm}$ and $\lambda_{\mathrm{em}}=668 \mathrm{~nm}$. Hydrodynamic diameters and

15 polydispersity index (PDI) were measured by dynamic light scattering (DLS, intensity-

16 average) using a Delsa Nano C particle analyzer (Beckman Coulter, Brea, CA)

17 (Supplementary Table 1).

\subsection{Immunoblot}

Whole protein lysates were generated by lysing the cells with Lysis buffer P [21].

Denatured whole protein lysates or concentrated supernatants $(30 \mu \mathrm{g} / \mathrm{lane})$ were separated on 10-15\% acrylamide gels. After transfer to nitrocellulose (Biorad, Hercules,

23 CA), blots were blocked in Tris-buffered saline with Tween ${ }^{\circledR} 20$ (TBST) (Sigma Aldrich) containing $5 \%$ milk and incubated overnight at $4{ }^{\circ} \mathrm{C}$ with primary antibodies specific for human (MT 23.2, ThermoFisher Scientific) or mouse (MAB3299, R\&D Systems, 
1 Cruz, CA). The membranes were then washed in TBST and incubated for $1 \mathrm{~h}$ at room

2 temperature with horseradish peroxidase (HRP)-coupled secondary antibodies: anti-

3 mouse, anti-rat or anti-goat (clones sc-2004 and sc-2033, Santa Cruz Biotechnologies).

4 Protein bands were visualized by enhanced chemiluminescence (ThermoFisher Scientific).

\section{$6 \quad$ 2.4. Clonogenic survival assays}

$7 \quad$ Clonogenic survival assays were performed by seeding 50 cells per well in a 96 -well plate

8 for SMA-497 cells. Cells were exposed to DMSO, O6 BTG-C18 dissolved in DMSO or LP-

$9 \mathrm{O}^{6} \mathrm{BTG}-\mathrm{C} 1824 \mathrm{~h}$ after seeding. Medium was removed $24 \mathrm{~h}$ later and TMZ was added at increasing concentrations. Growth of the SMA-497 cells was followed by observation for 15-20 days in FCS-containing medium. Cell density was assessed by crystal violet staining of adherent cells. Spherogenicity assays were performed by seeding $150 \mathrm{ZH}-161$ cells per well in a 96-well plate. Cells were exposed to DMSO, ${ }^{6}$ BTG-C18 dissolved in DMSO or LP-O ${ }^{6}$ BTG-C18 $24 \mathrm{~h}$ after seeding. TMZ was added at increasing concentrations and cell growth was followed by observation for 15-20 days. Sphere forming ability was assessed by the 3-(4,5-dimethylthiazol-2-yl)-2,5-diphenyltetrazolium bromide tetrazolium (MTT) cell proliferation assay for $\mathrm{ZH}-161$ cells.

\subsection{Hemolysis assay}

LP-O ${ }^{6}$ BTG-C18 was evaluated for its hemolytic activity on isolated human erythrocytes. Leukoreduced and filtered human erythrocytes derived from healthy donors were delivered in saline, adenine, glucose and mannitol (SAGM) nutrient solution and stored at $4{ }^{\circ} \mathrm{C}$ until usage. The hemolysis assay was performed by mixing equal volumes of erythrocyte suspension and free ${ }^{6}$ BTG-C18 dissolved in PBS containing polysorbate 80 (SigmaAldrich) (25\% v/v, isotonic) or LP-O ${ }^{6}$ BTG-C18 in PBS at final ${ }^{6}{ }^{B T G}-\mathrm{C} 18$ concentrations of either 31 or $310 \mu \mathrm{g} / \mathrm{mL}$. Hundred \% hemolysis was determined by preparing a sample of 
1 ultrapure water instead of liposomes. Additionally, control samples of PBS and Triton X-

$2100(10 \mu \mathrm{g} / \mathrm{ml})$ (Sigma-Aldrich) were mixed together with erythrocyte suspension as

3 negative and positive controls, respectively. The samples were incubated for $1 \mathrm{~h}$ at $37^{\circ} \mathrm{C}$

4 while gently shaking. Then, erythrocytes were separated by centrifugation at $1000 \times g$ for 5

5 min and the amount of released hemoglobin in the supernatant was determined by

6 spectrophotometry at $578 \mathrm{~nm}$ within a microtiter plate using an Infinite M200Pro plate

7 reader (Tecan).

\subsection{Cryo-transmission electron microscopy (cryo-TEM) imaging}

9 Samples of $5 \mu \mathrm{L}$ at a concentration of $4 \mathrm{~mol} \%$ were added to a copper grid covered by

10 holey carbon film (Quantifoil Multi A Micro Tools $\mathrm{GmbH}$, Jena, Germany). Each sample

11 was snap-frozen into a crybox of liquid ethane (Carl Zeiss NTS GmbH, Oberkochen,

12 Germany) and immidiatelly transferred into the pre-cooled cryo-electron microscope

13 (Philips CM 120, Eindhoven, Netherlands). Images were captured using a 2k CMOS

14 Camera (F216, TVIPS, Gauting, Germany) (Supplementary Fig. 1).

\subsection{Animal studies}

All procedures were performed in accordance with the Cantonal Veterinary Office Zurich and Swiss Federal Food Safety and Veterinary Office (Permission number 085/2014). For the studies involving glioma-bearing mice, VM/Dk mice were bred in house. Mice of 6-10 weeks of age were used in all experiments. Before all intracranial procedures, mice were anaesthetized using an intraperitoneal 3 component injection consisting of fentanyl (0.05 mg/kg; Fentanyl, Kantonsapotheke, Zurich), midazolam (5 mg/kg; Dormicum®, Roche Pharma AG, Reinach) and medetomidine (0.5 mg/kg; Dorbene ${ }^{\circledR}$, Graeub AG, Bern) mixed in $0.9 \% \mathrm{NaCl}$. For intracranial tumor cell implantation, the mice were fixed under a stereotactic device (Stoelting, Wood Dale, IL) and a burr hole was drilled into the skull 2 $\mathrm{mm}$ lateral and $1 \mathrm{~mm}$ posterior to the bregma. A Hamilton syringe needle was introduced 
1 to a depth of $3 \mathrm{~mm}$. SMA-497 murine glioma cells $\left(5 \times 10^{3}\right)$ in a volume of $2 \mu \mathrm{L}$ PBS were

2 injected into the right striatum of VM/Dk mice. For the in vivo MGMT depletion studies, 10

3 days after tumor inoculation, the LIFU protocol (section 2.7.2) was applied and animals

4 were perfused with PBS 48 or $72 \mathrm{~h}$ after treatment. Tumor-bearing and contralateral

5 hemispheres and liver were extracted. Tissues were dissociated with a scalpel and lysed

6 in RIPA lysis buffer containing protease (P-8340, Sigma) and phosphatase 2/3 (P-0044; P-

7 5726, Sigma) inhibitor cocktails. MGMT protein levels were assessed by immunoblot as

8 described in the 'Immunoblot section. For survival studies, 5 or 6 days after tumor

9 inoculation, the LIFU protocol was applied and 2 days later, TMZ (10 mg/kg) or DMSO control was orally administered for five consecutive days (Supplementary Fig. 2). TMZ was dissolved in sterile water containing $0.1 \%$ DMSO. Mice were sacrificed when they reached grade 2 neurological symptoms [22]. For hematological analyses, blood was collected from three pre-randomized mice three days after TMZ treatment and analyzed at the laboratory for veterinary medicine (Zurich, Switzerland). For liposome extravasation studies, the LIFU protocol was applied in healthy C57BL/6J (Charles River Laboratories, San Diego, CA) or VM/Dk mice intravenously injected with DiD-labeled liposomes (13 $\mu \mathrm{mol}$ lipid/kg) and $2 \mathrm{~h}$ after treatment animals were perfused with PBS and $20 \%$ paraformaldehyde (PFA). Brains were fixed for $24 \mathrm{~h}$ in $20 \%$ PFA and then cryoprotected in $30 \%$ sucrose solution for further $48 \mathrm{~h}$. Fixed brains were mounted in Cryochrome medium (ThermoFisher Scientific) and cut in sections of $8 \mu \mathrm{m}$. Processed brain sections were mounted on coverslips using the Dako fluorescent mounting medium (S3023, Agilent, Santa Clara, CA) and analyzed for DiD fluorescence (644/665 nm excitation/emission) and brain autofluoresence (430/490 $\mathrm{nm}$ excitation/emission) detection under the Axio Imager microscope (Zeiss, Oberkochen, Germany). Whole organ fluorescence imaging was performed using the CRi's Maestro In vivo Fluorescence Imaging System 2.2 (Cambridge Research \& Instrumentation, Inc., Woburn, MA). DiD fluorescence accumulation was quantified as DiD fluorescence signal 
1 ratios of the LIFU-treated brain regions versus the corresponding untreated regions of the

2 contralateral hemispheres using area fraction analyses [23] in the ImageJ analysis

3 software (https://imagej.nih.gov/ij/, Open Source). Ratio values of higher than 1 indicate

4 increased DiD fluorescence intensity/accumulation in the LIFU treated areas. Hematoxylin

5 and Eosin (H\&E) staining of cryosections was perfomed as previously [23]. For

6 immunofluorescence staining of blood vessels, cryosections were fixed, blocked, and

7 stained with primary antibody specific for CD31 (clone MEC 13.3, BD Biosciences,

8 Franklin Lakes, NJ), followed by the rabbit anti-rat IgG-Alexa Fluor 488-coupled

9 secondary antibody (Thermo Scientific, Waltham, MA) and mounted in Vectashield

10 Mounting Media with 4', 6'-diamino-2-phenylindole (DAPI) (Vector laboratories, Inc.,

11 Burlingame, CA). Confocal images were acquired using a Leica SP5 confocal

12 microscope (Wetzlar, Germany).

\subsubsection{Magnetic resonance imaging (MRI)}

Anesthesia was induced using $3 \%$ isoflurane (Abbott, Cham, Switzerland) in a 4:1 air/oxygen mixture. The tail vein was cannulated for administration of contrast agents. Body temperature was kept at $36.0 \pm 0.5^{\circ} \mathrm{C}$ using a hot water circuit integrated into the animal support (Bruker, Billerica, MA). Data were acquired on a Bruker BioSpec 47/30 (Bruker) small animal MR system operating at $4.7 \mathrm{~T}$. The system was equipped with a $2 \times 2$ phased-array mouse head surface coil (Bruker). Tumor size was measured using T2weighted MRI starting on day ten after tumor implantation, and then every three days with the following parameters: repetition time $(T R)=300 \mathrm{~ms}$, echo time $(T E)=3.854 \mathrm{~ms}$, matrix size $=384 \times 384$, field of view $(F O V)=20 \times 20 \mathrm{~mm}^{2}\left(\right.$ resolution $\left.=0.052 \times 0.052 \mathrm{~mm}^{2}\right)$, slice thickness $=0.5 \mathrm{~mm}$. Tumor volume was quantified using the Volumest plugin in the ImageJ analysis software (https://imagej.nih.gov/ij/, Open Source). BBB opening was confirmed by Gd-DOTA-enhanced T1-weighted MRI acquired with the following 
parameters: 3D FLASH sequence, $\mathrm{TR}=25 \mathrm{~ms}, \mathrm{TE}=3.884 \mathrm{~ms}$, flip angle $30^{\circ}$, number of average $=15$, matrix size $=40 \times 60 \times 40, \mathrm{FOV}=12 \times 20 \times 14 \mathrm{~mm}^{3}$ (resolution $=$ $0.300 \times 0.333 \times 0.350 \mathrm{~mm}^{3}$ ), Gd-DOTA $50 \mu \mathrm{l}$ intravenously (Guerbet, Paris, France). GdDOTA enhancement is shown as maximal intensity projection on a 3D-rendered mouse brain template using 3dSlicer (https://www.slicer.org, Open Source).

\subsubsection{MRI-guided low intensity-pulsed focused ultrasound (LIFU) application}

The intervention protocol for LIFU-mediated opening of the BBB on anesthetized mice consisted of animal preparation with clean shave of the skull region to be exposed to ultrasound, insertion of a tail vein catheter, positioning and fixation on an animal bed, acoustic coupling of skull and transducer and MRI-guided definition of target tissue volume. Mice were intravenously injected with $\mathrm{O}^{6} \mathrm{BTG}-\mathrm{C} 18$ or LP-O ${ }^{6} \mathrm{BTG}-\mathrm{C} 18$ at 7.2 $\mathrm{mg} / \mathrm{kg}$, corresponding to $310 \mu \mathrm{mol}$ lipid/kg. LIFU-mediated BBB permeabilization was achieved approximatelly 2 minutes after liposome administration, using a dedicated MRcompatible rodent FUS system (IGT, Pésac, France) attached to a 4.7 T rodent MRI system (Bruker) that allowed for computer-controlled positioning of the LIFU transducer and parametrization of LIFU application. The LIFU protocol consisted of standard isoflurane gas anesthesia and subsequent infusion of $50 \mu \mathrm{L}\left(16 \times 10^{6}\right.$ bubbles) softshell BG8235 microbubbles (Bracco Suisse SA) at a rate of $1 \mu \mathrm{L} / \mathrm{s}$ for a duration of $50 \mathrm{~s}$ in total. Thirty seconds after onset of the microbubble infusion, the right tumor-bearing hemisphere was sonicated at the tumor injection site as identified on T2-weighted MR images.

Ultrasound was applied using a 6-element annular array transducer (Imasonic, Besançon, France) carrying a passive cavitation detector and was delivered in bursts of $10 \mathrm{~ms}$ at a repetition frequency of $1 \mathrm{~Hz}$ for a total duration of $180 \mathrm{~s}$. LIFU pressure was controlled by an automated feedback loop that analyzed the passive cavitation detection (PCD) signatures and drove LIFU pressure below inertial cavitation threshold (0.28 - $0.55 \mathrm{MPa})$. 
Using real-time feedback from locally induced cavitation allowed to compensate for acoustic effects caused by physiological and geometrical differences between individual animals and setups (Supplementary Fig. 3). BBB opening was confirmed by gadolinium (Gd-DOTA)-enhanced (Sigma-Aldrich) MRI.

\subsubsection{Quantification of $O^{6} B T G-C 18$ in serum}

Blood was extracted 1, 3, and $6 \mathrm{~h}$ after tail vain injection of free or LP-O ${ }^{6} \mathrm{BTG}-\mathrm{C} 18$. At the indicated time, blood was collected via cardiac puncture in Microvette ${ }^{\circledR}$ tubes (Sarstedt, Sevelen, Switzerland) containing a clotting activator to obtain serum. The samples were gently mixed for $5 \mathrm{~min}$, left for $30 \mathrm{~min}$ to clot at room temperature and then centrifuged for 5 min at $10^{\prime} 000 \times g$. The supernatant was collected and immediately frozen in liquid nitrogen and stored at $-80{ }^{\circ} \mathrm{C}$ until further usage. Samples were spiked with an appropriate amount of the internal standard, $\mathrm{O}^{6} \mathrm{BTG}-\mathrm{C} 18(\mathrm{~N} 7) . \mathrm{O}^{6} \mathrm{BTG}-\mathrm{C} 18$ was purified via acetone protein precipitation followed by hexane liquid-liquid extraction and the drug amount was analyzed with liquid chromatography combined with a linear ion trap mass spectrometer (LC-MS) (LTQ XL, ThermoFisher Scientific) using a reverse-phase XBridge BEH C18 column (130 A, $5 \mu \mathrm{m}, 4.6 \mathrm{~mm} \times 250 \mathrm{~mm}$, Waters, Milford, MA). The employed method was isocratic using a methanol/water mixture of $97: 3(\mathrm{v} / \mathrm{v})$ and a flow rate of $0.8 \mathrm{~mL} / \mathrm{min}$. Drug quantification was carried out using calibration curves of spiked ${ }^{6}$ BTG-C18 standard solutions.

\subsection{Statistical analyses}

Statistical calculations were done using the GraphPad Prism Version 5 including paired ttest (single comparisons), 1-way ANOVA and Bonferroni post-hoc testing (multiple comparisons) and Log-rank (Martel-Cox) testing for survival analyses. $P$ values of $\leq 0.05$ $\left({ }^{*} ; \#\right), \leq 0.01\left({ }^{* *} ; \#\right)$ or $\leq 0.001\left(^{* * *}\right)$ were considered statistically significant. 
2 3. Results and Discussion

3 3.1. $\mathrm{O}^{6} \mathrm{BTG}-\mathrm{C} 18$ reduces MGMT protein levels and sensitizes glioma cells

4 to $T M Z$ exposure

5 The current standard of care for newly diagnosed glioblastoma involves tumor resection

6 followed by radiotherapy plus TMZ chemotherapy [2]. However, this regiment confers benefit

7 only to a small subgroup of patients with tumors harboring MGMT promoter methylation.

8 Therefore, there is a substantial need to overcome MGMT-mediated resistance and

9 increase the therapeutic activity of TMZ. Based on the commercially available MGMT

10 depleting drug $\mathrm{O}^{6} \mathrm{BTG}$, we synthesized several small molecule MGMT inactivators [17].

11 Through an initial screen of several first-in-class $0^{6} B T G$ derivatives we focused on the $12 \mathrm{O}^{6} \mathrm{BTG}-\mathrm{C} 18$ derivate with an N9 modification (Fig. 2A). $\mathrm{O}^{6} \mathrm{BTG}-\mathrm{C} 18$ reduced MGMT protein 13 levels in a concentration-dependent manner in murine SMA-497 and patient-derived $\mathrm{ZH}-$ 14161 glioma cells (Fig. 2B). In line with previous data [17, 24], substitution in the N9 position 15 did not substantially affect the MGMT-depleting activity of $\mathrm{O}^{6} \mathrm{BTG}$ in glioma cells. The 16 liposomal (LP)-O6 BTG-C18 nanoparticles had a mean hydrodynamic diameter of 17 approximately $72 \mathrm{~nm}$ (Supplementary Table 1). Cryo-TEM images of the vehicle liposomes 18 (control) and $\mathrm{LP}_{-} \mathrm{O}^{6} \mathrm{BTG}-\mathrm{C} 18$ confimed the spherical unilamellar structure of the nanoparticles [17] and indicated that no drug precipitate was present (Supplementary Fig. 1). We confirmed the biological activity of these stable LP-O ${ }^{6}$ BTG-C18 particles by their MGMT-depleting activity as soon as $24 \mathrm{~h}$ after exposure with the effect lasting up to $72 \mathrm{~h}$ post-treatment in glioma cells (Fig. 2C). MGMT depletion had the expected effect, as preexposure of free $\mathrm{O}^{6} \mathrm{BTG}-\mathrm{C} 18$ or $\mathrm{LP}-\mathrm{O}^{6} \mathrm{BTG}-\mathrm{C} 18$ sensitized SMA-497 or $\mathrm{ZH}-161$ cells to increasing TMZ concentrations; with the $\mathrm{EC}_{50}$ decreasing 6-fold for SMA-497 cells and 3fold for ZH-161 cells (Fig. 2D,E). Importantly, LP-O6 BTG-C18 did not exert any hemolytic effect on isolated human erythrocytes (Fig. 3), rendering this formulation suitable for further 
1 in vivo investigation. This is in contrast to the control formulation which had to be prepared

2 with a surfactant in order to dissolve $\mathrm{O}^{6} \mathrm{BTG}-\mathrm{C} 18$, which induced significant red blood cell

3 lysis in vitro.

4

A

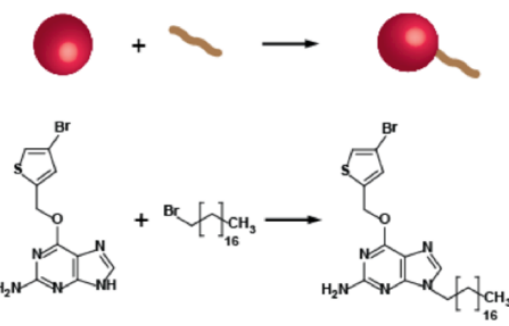

O ${ }^{6} \mathrm{BTG} \quad \mathrm{Br}-\mathrm{C} 18$

C

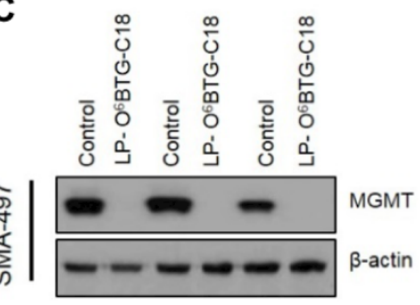

$\frac{\overline{0}}{\frac{1}{5}}$

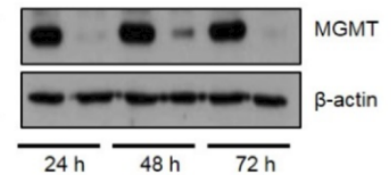

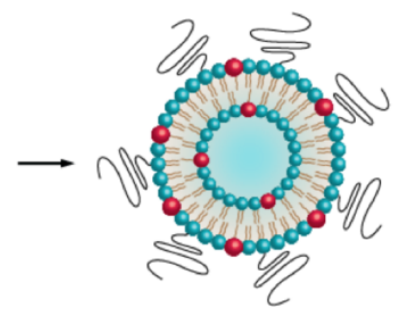

Liposomal-loaded (LP)-O ${ }^{6}$ BTG-C18

D
B

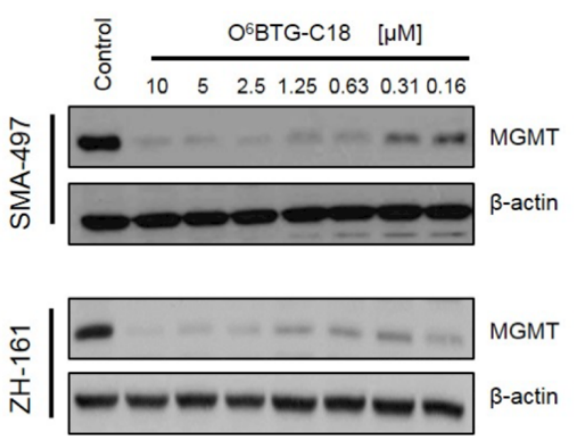

E

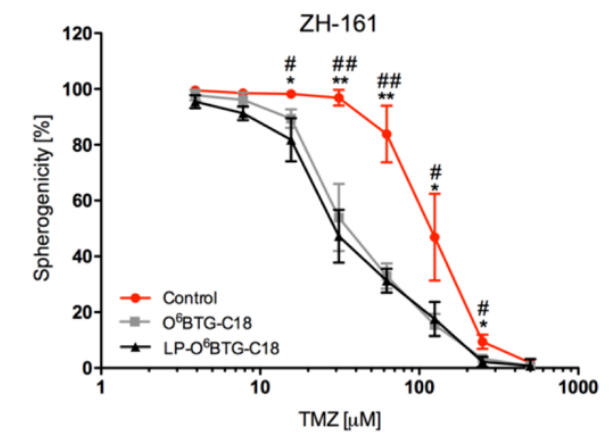

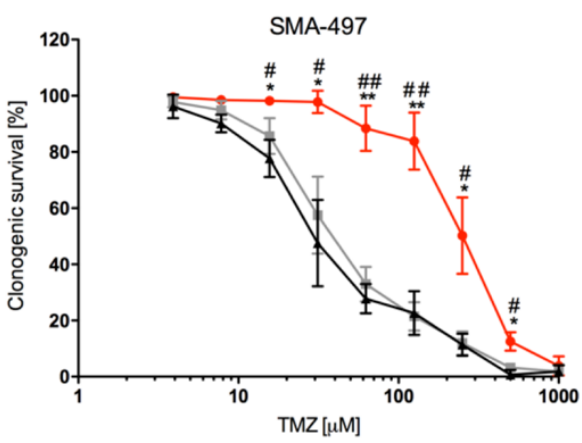

6 Figure 2. Free and liposomal $0^{6}$ BTG-C18 deplete MGMT in glioma cells. A. O6 BTGC18 was synthesized via nucleophilic substitution using bromoalkane $\mathrm{Br}-\mathrm{C} 18$ and lithium hydride as base in dimethylformamide at $80^{\circ} \mathrm{C}$. $75-\mathrm{nm}$ sized LP-O ${ }^{6} \mathrm{BTG}-\mathrm{C} 18$ was composed of 1,2-dioleoyl-sn-glycero-3-phosphocholine, cholesterol and 1,2-distearoyl-snglycero-3-phosphoethanolamine-poly(ethylene glycol) in a molar ratio of 72.5:20:7.5. B, C. Murine SMA-497 or patient-derived sphere-forming $\mathrm{ZH}-161$ glioma cells were exposed to increasing concentrations of free $\mathrm{O}^{6} \mathrm{BTG}-\mathrm{C} 18$ (B) or $5 \mu \mathrm{M}$ LP-O ${ }^{6}$ BTG-C18 (C) and MGMT protein levels were assessed by immunoblot.

D, E. SMA-497

(D) or $\mathrm{ZH}-161$ (E) cells were seeded for clonogenicity or spherogenicity assays and exposed to $5 \mu \mathrm{M}$ of $\mathrm{O}^{6} \mathrm{BTG}-\mathrm{C} 18$ or LP-O ${ }^{6}$ BTG-C18 for $24 \mathrm{~h}$. TMZ was added at increasing concentrations and the cells were allowed to grow for 20 days. Clonogenicity or spherogenicity was determined by crystal 
1 violet or MTT staining, respectively. * = DMSO vs ${ }^{6}{ }^{6} \mathrm{BTG}-\mathrm{C} 18$; \# = DMSO vs LP-O ${ }^{6} \mathrm{BTG}-$

2 C18. Results are expressed as means and SD $(n=3)$, statistical analysis was performed

3 with one-way ANOVA and Bonferroni post-hoc testing with * or \# $p \leq 0.05$, ${ }^{* *}$ or \#\# $p \leq$

$4 \quad 0.01$ and ${ }^{* * *} p \leq 0.001$.

5

6 Figure 3. LP-O ${ }^{6} \mathrm{BTG}-\mathrm{C} 18$ does not induce hemolysis of human erythrocytes. $\mathrm{O}^{6} \mathrm{BTG}-$

7 C18 dissolved in polysorbate 80 solution or LP-O ${ }^{6}$ BTG-C18 were mixed together with

8 human erythrocytes in suspension for $1 \mathrm{~h}$ at $37^{\circ} \mathrm{C}$. Released hemoglobin in the

9 supernatant was determined by spectrophotometry. Triton X-100 was used as positive

control, PBS as a negative control, and polysorbate 80 or empty LP vehicles were used as controls for their respective agents. Hundred \% of hemolysis threshold was identified using ultrapure water. Results are expressed as means and SD $(n=3)$.

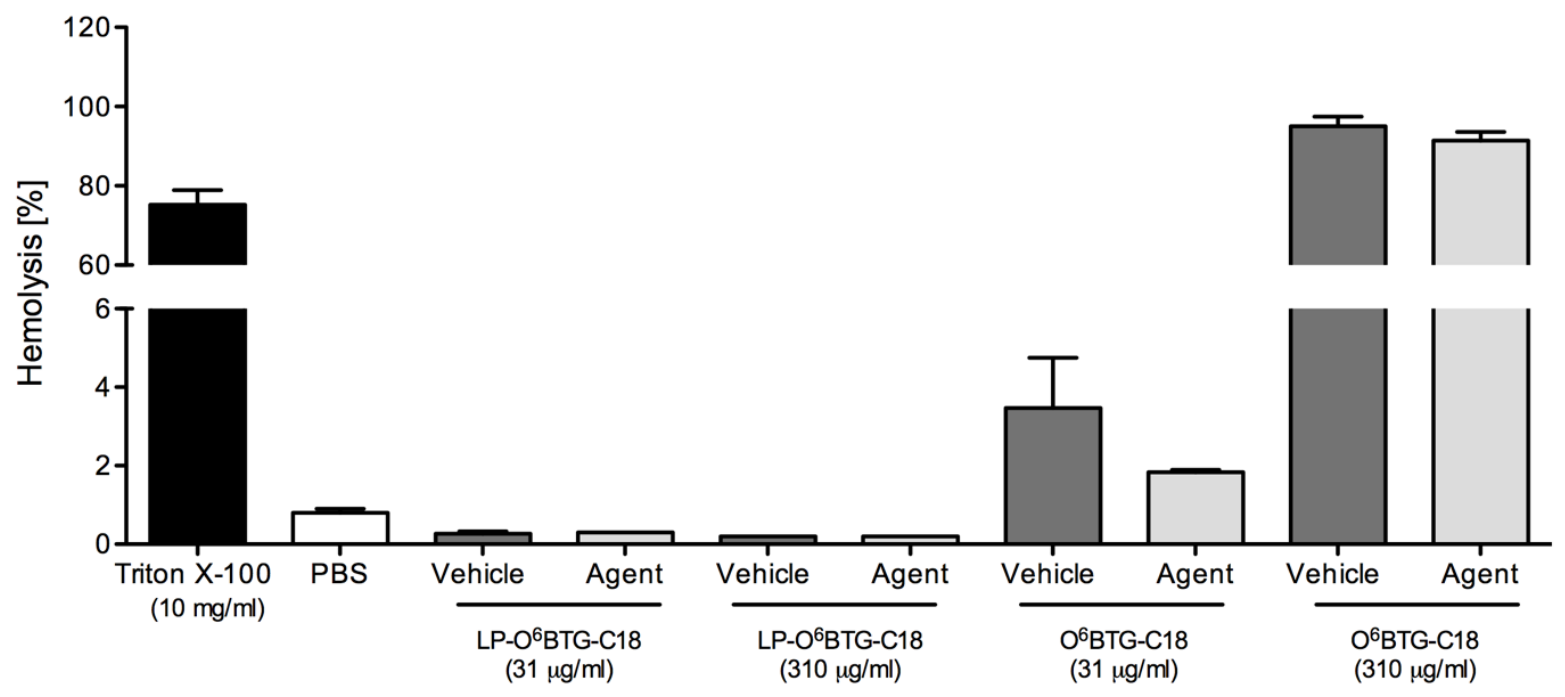


3.2. MRI-guided LIFU facilitates BBB opening

3 We developed a LIFU protocol to deliver the loaded liposomes across the BBB in mice

4 using a dedicated experimental platform that allows for non-invasive, MRI-guided, fast,

5 and reproducible BBB opening. Ultrasound pressure was controlled by an automated

6 feedback loop that analyzed the passive cavitation detection signatures and drove LIFU

7 pressure below inertial cavitation threshold $(0.28-0.55 \mathrm{MPa})$. Our LIFU system performed

8 an in situ titration of the acoustic pressure for each animal prior to the application of the

9 therapeutic pulses. Based on the determined target cavitation dose, a microbubble feedback loop ensured that ultrasound pulses were applied to reliably open the BBB without causing irreversible damage to the brain tissue (Supplementary Fig. 3). This protocol differs from traditional methods [12, 25-27], since it does not titrate pressure through a cohort of test animals to establish a fixed acoustic pressure, but instead determines a safe and adequate pressure for each animal individually using a softshell microbubble feedback loop. Using this novel approach we could compensate for acoustic effects caused by physiological and geometrical differences between animals and experimental conditions. Common techniques to confirm ultrasound-mediated BBB opening include contrastenhanced T1-weighted MRI, assessment of extravasated Evans blue dye [15], or histological detection of the delivered drug agent [15]. Here, besides conducting in vivo gadolinium (Gd-DOTA)-enhanced MRI to confirm BBB opening (Fig. 4A), we generated and delivered stable fluorescent lipophilic carbocyanine derivative (DiD)-labelled liposomes immediately after LIFU application. DiD-labelled liposomes were visualized ex vivo $2 \mathrm{~h}$ after LIFU treatment, whereas Gd-DOTA enhancement MRI was performed in real-time right after the LIFU-mediated BBB opening. Together with the strong positive contrast enhancement and rapid body elimination, the smaller size of Gd-DOTA [28] might explain the more pronounced extravasation observed compared to DiD-labelled liposomes 
1 (Fig. 4A). Similar to quantum dot-loaded cationic liposomes [29], DiD-labelled liposomes

2 were detected ex vivo specifically in the sonication area (Fig. 4B-E). In agreement with

3 previous studies, blood vessel staining revealed no apparent damage on vasculature

4 structure after LIFU treatment (Supplementary Fig. 4), suggesting that LIFU does not

5 cause irreversible damage to the BBB [11, 12]. Quantification of DiD fluorescence in the

6 sonicated brain areas versus untreated regions of the contralateral hemispheres revealed

7 significantly increased levels of DiD-liposome accumulation in the LIFU-treated region (Fig.

8 4F). These data demonstrate that controlled LIFU-mediated BBB disruption can efficiently

9 and selectively facilitate the delivery of liposomal formulations into the target regions under

10 MRI guidance.
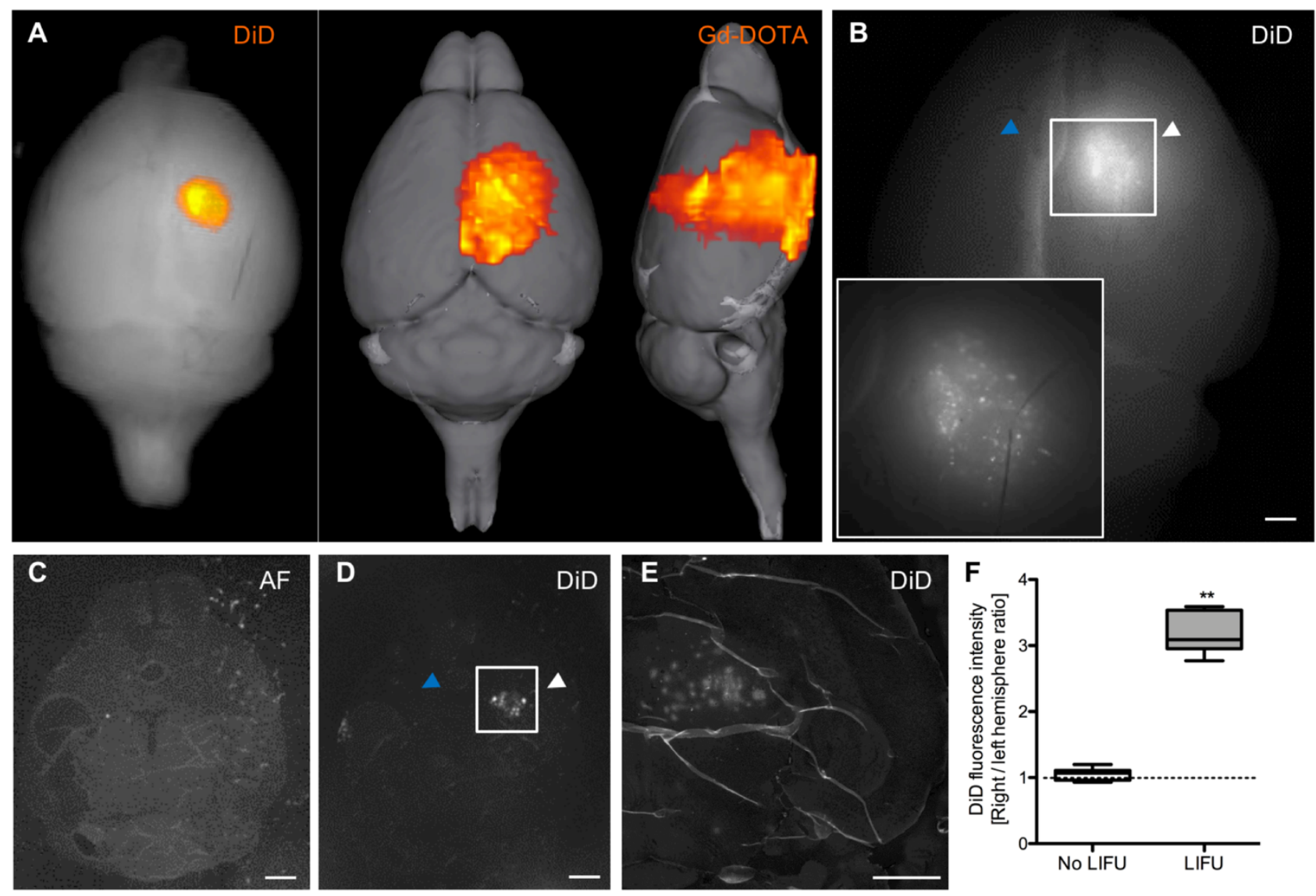

15 Figure 4 legend on next page. 
1 Figure 4. LIFU-mediated BBB opening facilitates liposomal nanoparticle delivery. A-

2 F. Healthy mice were intravenously injected with DiD-labelled liposomes $(0.3 \mu \mathrm{g} / \mathrm{kg})$. LIFU

3 was applied targeting a specific region in the right hemisphere (white arrowheads) with the

4 contralateral non-LIFU treated hemisphere serving as a control (blue arrowheads). 3D

5 reconstruction of enhancing region in T1-weighted MRI after intravenous administration of

6 Gd-DOTA following LIFU application overlaid to schematic mouse brain is shown. The

7 color-coded region indicates the extent of Gd-DOTA extravasation due to LIFU-mediated

8 BBB disruption. DiD fluorescence and enhancing MRI volumes are depicted (A). DiD

9 extravasation at the sonication site was assessed on perfused brains ex vivo either macroscopically (B) or using histological sections (C-E) by fluorescence microscopy. The 430/490 $\mathrm{nm}$ excitation/emission was used as a control for brain autofluoresence (AF).

White squares depict the location of sonication and DiD-labelled LP extravasation. Scale bar $=200 \mu \mathrm{m} .10 x$ magnification in white square $(B, E)$. Representative images of one mouse $(n=4)$. F. DiD fluorescence accumulation was quantified as DiD fluorescence intensity ratio of the LIFU-treated or non-LIFU exposed brain regions of the right hemisphere versus the corresponding untreated regions of the contralateral hemisphere. The dashed line indicates the baseline ratio for no change. Results are expressed as means and SD $(n=4)$, statistical analysis was performed with paired t-test with ${ }^{* *} p \leq 0.01$. 


\section{3. $L P-O^{6} B G T-C 18$ in combination with LIFU exerts potent in vivo MGMT-depleting} activity in glioma-bearing mice

Next, we assessed the pharmacokinetics and in vivo activity of LP-O ${ }^{6}$ BTG-C18 in SMA497 glioma-bearing mice after intravenous application. Liposomal entrapment resulted in a substantial reduction in the clearance of $\mathrm{O}^{6} \mathrm{BTG}-\mathrm{C} 18$, corresponding to an almost 20 -fold increase in area under the serum concentration versus time curve, up to $6 \mathrm{~h}$ post-injection compared to the control formulation (Fig. 5A). We then went on to assess degradationinducing MGMT protein depletion after systemic administration of LP-O6 BTG-C18 in SMA497 glioma-bearing mice. The increase in systemic exposure combined with an increased delivery across the LIFU-permeabilized BBB allowed for a potent reduction in MGMT protein levels 48 and $72 \mathrm{~h}$ after treatment in orthotopically growing gliomas (Fig. 5B). Free $\mathrm{O}^{6} \mathrm{BTG}-\mathrm{C} 18$ reduced MGMT protein levels after LIFU treatment only after $72 \mathrm{~h}$ (Fig. 5B). This less prominent effect for the ${ }^{6}{ }^{6} \mathrm{BTG}-\mathrm{C} 18$ control formulation indicates that a prolonged circulation time is important for sufficient deposition of the agent at the tumor site. Moreover, the fact that LP-O ${ }^{6} \mathrm{BTG}-\mathrm{C} 18$ required $48 \mathrm{~h}$ to exert its potent MGMT depleting activity after assisted LIFU guidance into the tumor area suggests that a potential sustained ${ }^{6}$ BTG-C18 release from the liposomal formulation might be in effect. Neither agents formulated with liposomes nor in solution had an effect on MGMT protein levels without LIFU application (Fig. 5B), indicating that BBB opening is required to enhance their activity at the tumor site. Our data are in line with other therapeutic approaches attempting to reduce MGMT protein levels in TMZ-resistant gliomas via the delivery of MGMT-targeting siRNA oligonucleotides either by continuous administration using osmotic pumps [30], or direct tumoral injections [31]. However, our approach has the advantage of utilizing a derivative of an MGMT inactivator combined with a non-invasive delivery method already considered for clinical use [7, 8]. Liver MGMT levels were used as additional control and remained unaltered after treatment (Fig. 5C), suggesting that the 
1 sonication of the tissue in the brain area may facilitate the diffusion and cellular uptake of

2 LP-O ${ }^{6}$ BTG-C18. Moreover, colloidal formulations (control micelles and liposomes) are

3 known to be mainly taken up by the mononuclear phagocyte system rather than

4 hepatocytes [32].

5

A

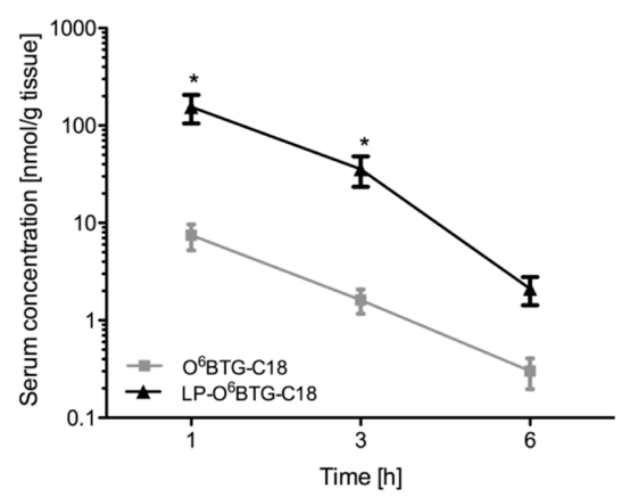

B Tumor hemisphere

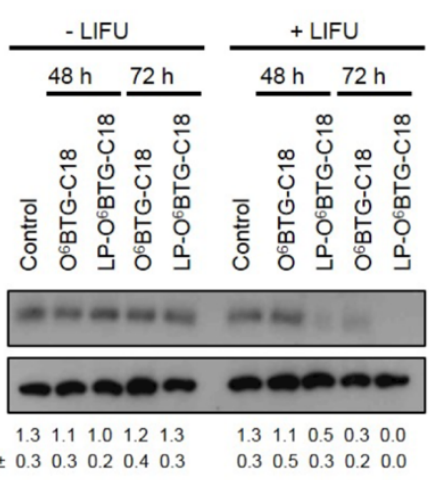

C Liver
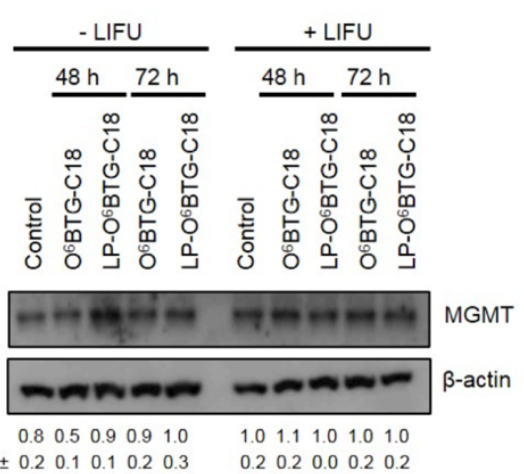

6

Figure 5. LP-O ${ }^{6}$ BTG-C18 depletes tumoral MGMT levels after LIFU-mediated delivery in glioma-bearing mice. A-C. Five $\times 10^{3}$ SMA-497 glioma cells were inoculated

stereotactically into the right hemisphere of VM/Dk mice. Ten days after tumor inoculation, mice were intravenously injected with free $\mathrm{O}^{6} \mathrm{BTG}-\mathrm{C} 18$ or LP-O ${ }^{6} \mathrm{BTG}-\mathrm{C} 18$ (dose for both formulations: $7.2 \mathrm{mg} / \mathrm{kg}$ ). Mice were PBS-perfused 1, 3, or $6 \mathrm{~h}$ after treatment and serum was collected for determination of $\mathrm{O}^{6} \mathrm{BTG}-\mathrm{C} 18$ concentration via liquid chromatographymass spectrometry. Results are expressed as means and SD $(n=3-6)$. Statistical analysis was performed with one-way ANOVA and Bonferroni post-hoc testing with ${ }^{*} p<0.05$ (A). The tumor region was targeted using MRI and the LIFU sonications were either applied (+LIFU) or not (-LIFU). Mice were PBS-perfused 48 or $72 \mathrm{~h}$ after treatment and the tumorbearing hemisphere $(n=3)$ or liver $(n=2)$ were lysed. MGMT protein levels in brain $(B)$ and liver (C) samples were assessed by immunoblot. Densitometry analyses of MGMT protein levels normalized to the corresponding $\beta$-actin control are depicted. Vehicle injections were used as a control. Results are expressed as means and SD. 
2 3.4. LIFU-mediated delivery of $L P-\mathrm{O}^{6} B T G-C 18$ reduces tumor growth in vivo and

3 prolongs survival of mice bearing TMZ-resistant experimental gliomas

4 Based on these promising findings, we went on to examine the combination of LP-O ${ }^{6} \mathrm{BTG}-$

$5 \quad$ C18 and LIFU as a strategy for TMZ sensitization in vivo. Male and female mice were

6 distributed equally amongst the treatment groups. The tumor region in the right hemisphere

7 of SMA-497 glioma-bearing mice was targeted for focused LIFU sonication under MRI

8 guidance and Gd-DOTA-enhanced T1-weighted MRI confirmed the BBB opening (Fig. 6A).

9 Our data are in agreement with previous studies [14, 15, 33], showing enhanced Evans blue

or gadolinium extravasation after ultrasound sonication in the tumor region, compared to the leaky glioma vasculature itself (Supplementary Fig. 5). LP-O6 BTG-C18 was administered intravenously immediately before LIFU application and subsequent oral treatment with TMZ significantly reduced the growth rate of SMA-497 gliomas as demonstrated by MRI (Fig. 6B,C), and prolonged survival compared to all other treatment groups (Fig. 6D; Supplementary Table 2). In contrast to previous studies [17, 18, 26, 28], our experimental setup allowed to control for the extent of LIFU-mediated BBB opening between the treatment groups, as visualized by Gd-DOTA influx (Supplementary Fig. 6). The comparable grades of BBB opening between the groups receiving LIFU treatment suggest that the survival prolongation can be attributed to the combinatorial treatment regimen and not any potential variation in BBB openings. While a single LIFU-mediated LP-O ${ }^{6}$ BTG-C18 dose was sufficient to sensitize gliomas to $\mathrm{TMZ}$, tumor growth inhibition could potentially be propagated by frequent treatments. The pronounced therapeutic efficacy of this novel combinatorial treatment was confirmed in a second, independent study (Supplementary Fig. 7). LIFU in combination with $\mathrm{LP}-\mathrm{O}^{6} \mathrm{BTG}-\mathrm{C} 18$ and $\mathrm{TMZ}$ represents the first treatment approach resulting in a clear therapeutic benefit in this highly therapy-resistant glioma model. Experimental SMA-497 gliomas closely recapitulate the highly infiltrative nature of 
1 human glioblastoma, and respond neither to radiotherapy nor anti-angiogenic treatment nor

2 transforming growth factor (TGF)- $\beta$ receptor inhibition [23]. Given their aggressive growth in

3 vivo, previous studies have failed to show a survival benefit upon treatment with various

4 drugs $[23,34]$. Since LP-O ${ }^{6}$ BTG-C18 is biologically active as an MGMT inactivator in human

$5 \mathrm{ZH}-161$ glioma cells as well (Fig. 2C,E), assessing the efficacy of this combinatorial

6 approach in patient-derived xenograft glioma models can further aid its clinical translation.

7 Other studies showed that concurrent TMZ administration and ultrasound-induced BBB 8 disruption prolonged the survival of animals bearing MGMT promoter-methylated gliomas

$9[14,15]$. In our experiments, mice treated with LIFU and TMZ also exhibited reduced tumor

10 growth and prolonged survival compared to TMZ alone despite the inherent MGMT-

11 mediated TMZ resistance of SMA-497 gliomas (Fig. 6 C,D; Supplementary Table 2;

12 Supplementary Fig. 7). Although dose-dense treatments did not demonstrate improved TMZ 13 efficacy in patients with glioblastomas expressing MGMT [35], our findings suggest that 14 ultrasound-mediated BBB opening could increase TMZ exposure and anti-tumor activity 15 [15]. Previous studies have shown that TMZ has the potential to deplete MGMT when 16 administered in higher concentrations [36, 37], which may explain the marginal survival 17 benefit observed with TMZ treatment when LIFU was applied without the liposomal MGMT inactivator in our model. We did not observe acute hematological toxicity or other side effects when ${ }^{6}{ }^{6}$ TTG-C18 was applied as a liposomal formulation together with TMZ or combined with transient BBB opening or both. The overall white blood cell, red blood cell and platelet count remained unchanged within normal ranges (Supplementary Fig. 8). Since LP-O6BBTGC18 was given intravenously to glioma-bearing mice and TMZ was administered orally, our study also provides a better model of the clinical setting compared to previous approaches $[38,39]$. Compared to our study, an almost 5 -fold higher dose of $0^{6} \mathrm{BTG}$ was required in other experimental set-ups to sensitize other tumors to alkylating chemotherapy [40, 41]. 
1 The majority of studies assessing the penetration and efficacy of therapeutic nanoparticles

2 in pre-clinical glioma models utilize relatively large liposomes that largely rely on passive

3 BBB delivery via the enhanced permiability and retention effect [30, 31, 42, 43]. The data

4 presented in this study strongly suggest that $\mathrm{O}^{6} \mathrm{BTG}-\mathrm{C} 18$-loaded liposomes require LIFU

5 application to efficiently bypass the BBB and exert potent chemosensitizing effects.

6 Alternative ways to circumvent the BBB assessed in the clinical setting include direct local

7 delivery of chemotherapeutic agents in the form of biodegradable polymer wafers at the time

8 of surgery [44], or the use of convection-enhanced delivery via intra-parenchymal micro-

9 catheters [45]. However, these applications carry severe limitations compared to the LIFU-

10 based approach, including invasive surgical placement, the increased risk of local neuro-

11 toxicity, hemorrhage, edema, or infection as well as the limited penetration of the drug [46]. 
A Pre-LIFU

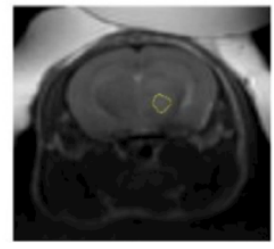

B

Untreated

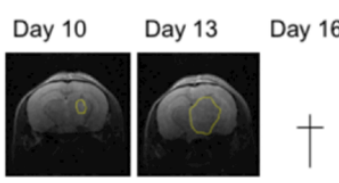

LIFU
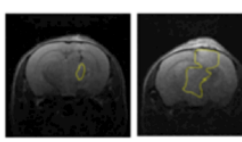

TMZ
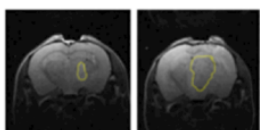

LP-O6BTGC18

TMZ+LP-

O6BTG-C18
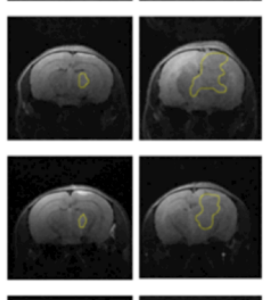

LIFU+TMZ
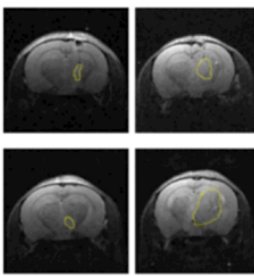

LIFU+TMZ+ LP-O'BTG C18

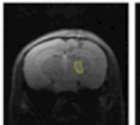

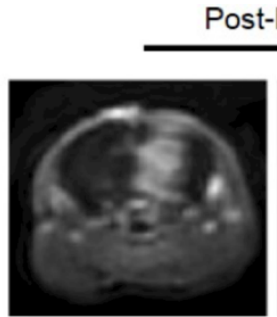

Day 19
Day 28

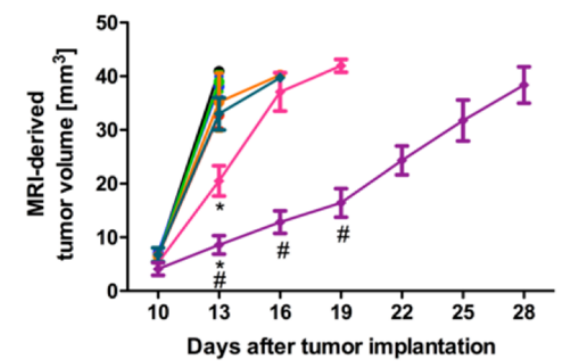

D
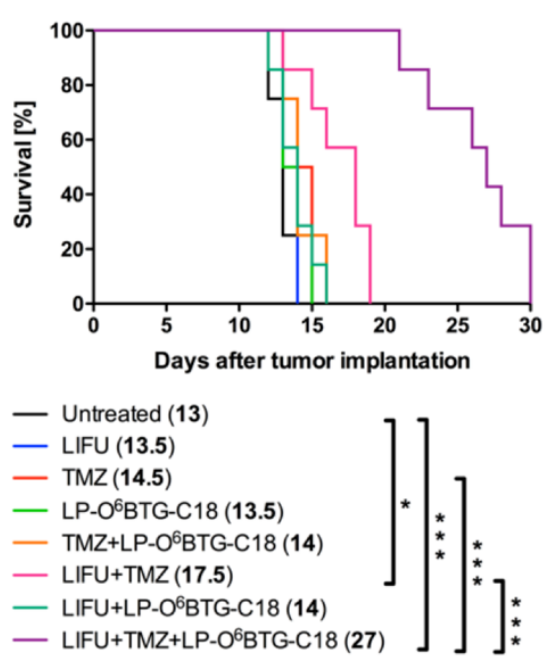

4 Figure 6. LIFU in combination with systemic LP-O6 BTG-C18 and TMZ treatment

5 reduces tumor growth and prolongs survival of glioma-bearing mice. A-D. Five $\times 10^{3}$

6 SMA-497 glioma cells were injected stereotactically into the right hemisphere of VM/Dk

7 mice. Six days after tumor implantation the LIFU protocol was applied targeting the tumor

8 area via MRI together with $\mathrm{LP}-\mathrm{O}^{6} \mathrm{BTG}-\mathrm{C} 18$ or control treatment. BBB opening was

9 visualized by T1-weighted Gd-DOTA-enhanced MRI (A). Two days later systemic oral

10 administration with $\mathrm{TMZ}(10 \mathrm{mg} / \mathrm{kg})$ or DMSO control was initiated for five consecutive

11 days. B, C. T2-weighted MRI tumor volume monitoring was initiated ten days after tumor 
1 inoculation and then every three days for three pre-randomized animals. Representative

$2 \mathrm{MRI}$ images of one mouse per group are shown with the yellow lines depicting the tumor

3 area $(n=3)$. Crosses $(\dagger)$ indicate that the animals had to be sacrificed due to score 2

4 neurological symptoms. Values are expressed as means and SD $(n=3)$, statistical analysis

5 was performed with one-way ANOVA and Bonferroni post-hoc testing with * or \# $p<0.05$.

$6 \quad{ }^{*}=\mathrm{TMZ}$ vs LIFU+TMZ or LIFU+TMZ+LP-O ${ }^{6} \mathrm{BTG}-\mathrm{C} 18 ;$ \# = LIFU+TMZ vs LIFU+TMZ+LP-

$7 \quad \mathrm{O}^{6} \mathrm{BTG}-\mathrm{C}$ 18. D. Mice were treated as described in (A) and sacrificed when they developed

8 grade 2 neurological symptoms ( $n=4-7$ per group). Kaplan-Meier survival curves are

9 shown with median survival depicted in parentheses for each group. Survival curves were 10 analyzed for differences via Log-rank (Martel-Cox) test with ${ }^{*} p<0.05$ or ${ }^{* * *} p<0.001$. of non-invasive modalities towards clinical application for brain diseases with an unmet medical need. 


\section{Appendix A. Supplementary data}

2 Supplementary data to this article are available online.

3

\section{Acknowledgments}

5 The work was supported by a Sinergia grant from the Swiss National Science Foundation

6 (SNSF, CRSII3_147651 to JCL, EM and MW). We thank Markus Marks from

7 Neurotechnology Group at ETH Zurich and Franziska Wiget from the Laboratory for

8 Molecular Neuro-Oncology at University Hospital Zurich for the technical support.

9

10

11

Conflict of Interest

13

The authors declare no conflict of interest.

Highlights

- A novel therapeutic liposomal agent inactivates MGMT in glioblastoma

- MRI-guided LIFU opens the BBB to deploy nanotherapeutics in targeted regions

- LIFU-driven delivery of liposomal MGMT inactivators reverses glioma 
[1] Q.T. Ostrom, H. Gittleman, J. Fulop, M. Liu, R. Blanda, C. Kromer, Y. Wolinsky, C.

Kruchko, J.S. Barnholtz-Sloan, CBTRUS statistical report: primary brain and central nervous system tumors diagnosed in the United States in 2008-2012, Neuro Oncol, 17 Suppl 4 (2015) iv1-iv62.

[2] M. Weller, M. van den Bent, J.C. Tonn, R. Stupp, M. Preusser, E. Cohen-JonathanMoyal, R. Henriksson, E. Le Rhun, C. Balana, O. Chinot, M. Bendszus, J.C. Reijneveld, F. Dhermain, P. French, C. Marosi, C. Watts, I. Oberg, G. Pilkington, B.G. Baumert, M.J.B.

Taphoorn, M. Hegi, M. Westphal, G. Reifenberger, R. Soffietti, W. Wick, European Association for Neuro-Oncology (EANO) guideline on the diagnosis and treatment of adult astrocytic and oligodendroglial gliomas, Lancet Oncol, 18 (2017) e315-e329.

[3] R. Stupp, W.P. Mason, M.J. van den Bent, M. Weller, B. Fisher, M.J.B. Taphoorn, K. Belanger, A.A. Brandes, C. Marosi, U. Bogdahn, J. Curschmann, R.C. Janzer, S.K. Ludwin, T. Gorlia, A. Allgeier, D. Lacombe, J.G. Cairncross, E. Eisenhauer, R.O. Mirimanoff, D. Van Den Weyngaert, S. Kaendler, P. Krauseneck, N. Vinolas, S. Villa, R.E. Wurm, M.H.B. Maillot, F. Spagnolli, G. Kantor, J.P. Malhaire, L. Renard, O. De Witte, L. Scandolaro, C.J. Vecht, P. Maingon, J. Lutterbach, A. Kobierska, M. Bolla, R. Souchon, C. Mitine, T. Tzuk-Shina, A. Kuten, G. Haferkamp, J. de Greve, F. Priou, J. Menten, I. Rutten, P. Clavere, A. Malmstrom, B. Jancar, E. Newlands, K. Pigott, A. Twijnstra, O. Chinot, M. Reni, A. Boiardi, M. Fabbro, M. Campone, J. Bozzino, M. Frenay, J. Gijtenbeek, A.A. Brandes, J.Y. Delattre, U. Bogdahn, U. De Paula, M.J. van den Bent, C. Hanzen, G. Pavanato, S. Schraub, R. Pfeffer, R. Soffietti, M. Weller, R.D. Kortmann, M. Taphoorn, J.L. Torrecilla, C. Marosi, W. Grisold, P. Huget, P. Forsyth, D. Fulton, S. Kirby, R. Wong, D. Fenton, B. Fisher, G. Cairncross, P. Whitlock, K. Belanger, S. Burdette-Radoux, S. Gertler, S. Saunders, K. Laing, J. Siddiqui, L.A. Martin, S. Gulavita, J. Perry, W. Mason, B. Thiessen, H. Pai, Z.Y. Alam, D. Eisenstat, W. Mingrone, S. Hofer, G. Pesce, J. Curschmann, P.Y. Dietrich, R. Stupp, R.O. Mirimanoff, P. Thum, B. Baumert, G. Ryan, E.O.R.T.C. Br, Radiotherapy plus concomitant and adjuvant temozolomide for glioblastoma, New Engl J Med, 352 (2005) 987-996.

[4] M.E. Hegi, A. Diserens, T. Gorlia, M. Hamou, N. de Tribolet, M. Weller, J.M. Kros, J.A. Hainfellner, W. Mason, L. Mariani, J.E.C. Bromberg, P. Hau, R.O. Mirimanoff, J.G. Cairncross, R.C. Janzer, R. Stupp, MGMT gene silencing and benefit from temozolomide in glioblastoma, New Engl J Med, 352 (2005) 997-1003. 
[5] M. Weller, R. Stupp, G. Reifenberger, A.A. Brandes, M.J. van den Bent, W. Wick, M.E. Hegi, MGMT promoter methylation in malignant gliomas: ready for personalized medicine?, Nature Rev. Neurology, 6 (2010) 39-51.

[6] M. Esteller, J.G. Herman, Generating mutations but providing chemosensitivity: the role of O6-methylguanine DNA methyltransferase in human cancer, Oncogene, 23 (2004) 1-8. [7] J.A. Quinn, S.X. Jiang,

D.A. Reardon, A. Desjardins, J.J. Vredenburgh, J.N. Rich, S. Gururangan, A.H. Friedman, D.D. Bigner, J.H. Sampson, R.E. McLendon, J.E. Herndon, A. Walker, H.S. Friedman, Phase II trial of temozolomide plus $\mathrm{O}(6)$-benzylguanine in adults with recurrent, temozolomide-resistant malignant glioma, J Clin Oncol, 27 (2009) 1262-1267.

[8] H.A. Tawbi, L. Villaruz, A. Tarhini, S. Moschos, M. Sulecki, F. Viverette, J. ShipeSpotloe, R. Radkowski, J.M. Kirkwood, Inhibition of DNA repair with MGMT pseudosubstrates: phase I study of lomeguatrib in combination with dacarbazine in patients with advanced melanoma and other solid tumours, Br J Cancer, 105 (2011) 773777.

[9] O. van Tellingen, B. Yetkin-Arik, M.C. de Gooijer, P. Wesseling, T. Wurdinger, H.E. de Vries, Overcoming the blood-brain tumor barrier for effective glioblastoma treatment, Drug Resist Update, 19 (2015) 1-12.

[10] N. Sheikov, N. McDannold, N. Vykhodtseva, F. Jolesz, K. Hynynen, Cellular mechanisms of the blood-brain barrier opening induced by ultrasound in presence of microbubbles, Ultrasound Med Biol, 30 (2004) 979-989.

[11] N. McDannold, N. Vykhodtseva, K. Hynynen, Blood-brain barrier disruption induced by focused ultrasound and circulating preformed microbubbles appears to be characterized by the mechanical index, Ultrasound Med Biol, 34 (2008) 834-840.

[12] K. Hynynen, N. McDannold, N. Vykhodtseva, F.A. Jolesz, Noninvasive MR imagingguided focal opening of the blood-brain barrier in rabbits, Radiology, 220 (2001) 640-646. [13] L.H. Treat, N. McDannold, N. Vykhodtseva, Y. Zhang, K. Tam, K. Hynynen, Targeted delivery of doxorubicin to the rat brain at therapeutic levels using MRI-guided focused ultrasound, Int J Cancer, 121 (2007) 901-907.

[14] K.-C. Wei, P.-C. Chu, H.-Y.J. Wang, C.-Y. Huang, P.-Y. Chen, H.-C. Tsai, Y.-J. Lu, P.Y. Lee, I.C. Tseng, L.-Y. Feng, P.-W. Hsu, T.-C. Yen, H.-L. Liu, Focused ultrasoundinduced blood-brain barrier opening to enhance temozolomide delivery for glioblastoma treatment: a preclinical study, Plos One, 8 (2013) e58995. 
[15] H.L. Liu, C.Y. Huang, J.Y. Chen, H.Y. Wang, P.Y. Chen, K.C. Wei, Pharmacodynamic

and therapeutic investigation of focused ultrasound-induced blood-brain barrier opening for enhanced temozolomide delivery in glioma treatment, PLoS One, 9 (2014) e114311. [16] J. Park, M. Aryal, N. Vykhodtseva, Y.Z. Zhang, N. McDannold, Evaluation of permeability, doxorubicin delivery, and drug retention in a rat brain tumor model after ultrasound-induced blood-tumor barrier disruption, J Control Release, 250 (2017) 77-85. [17] R.D. Signorell, A. Papachristodoulou, J. Xiao, B. Arpagaus, T. Casalini, J. Grandjean, J. Thamm, F. Steiniger, P. Luciani, D. Brambilla, B. Werner, E. Martin, M. Weller, P. Roth, J.C. Leroux, Preparation of PEGylated liposomes incorporating lipophilic lomeguatrib derivatives for the sensitization of chemo-resistant gliomas, Int J Pharm, 536 (2018) 388396.

[18] R.D. Serano, C.N. Pegram, D.D. Bigner, Tumorigenic cell culture lines from a spontaneous VM/Dk murine astrocytoma (SMA), Acta neuropathologica, 51 (1980) 53-64. [19] D. Lemke, M. Weiler, J. Blaes, B. Wiestler, L. Jestaedt, A.C. Klein, S. Low, G. Eisele, B. Radlwimmer, D. Capper, K. Schmieder, M. Mittelbronn, S.E. Combs, M. Bendszus, M. Weller, M. Platten, W. Wick, Primary glioblastoma cultures: can profiling of stem cell markers predict radiotherapy sensitivity?, J Neurochem, 131 (2014) 251-264.

[20] M. Schneider, B. Anantharam, M. Arditi, D. Bokor, A. Broillet, P. Bussat, X. Fouillet, P. Frinking, I. Tardy, J. Terrettaz, R. Senior, F. Tranquart, BR38, a new ultrasound blood pool agent, Invest Radiol, 46 (2011) 486-494.

[21] K. Seystahl, A. Papachristodoulou, I. Burghardt, H. Schneider, K. Hasenbach, M. Janicot, P. Roth, M. Weller, Biological role and therapeutic targeting of TGF- $\beta 3$ in glioblastoma, Mol Cancer Ther, 16 (2017) 1177-1186.

[22] E. Szabo, H. Schneider, K. Seystahl, E.J. Rushing, F. Herting, K.M. Weidner, M. Weller, Autocrine VEGFR1 and VEGFR2 signaling promotes survival in human glioblastoma models in vitro and in vivo, Neuro Oncol, 18 (2016) 1242-1252.

[23] D. Mangani, M. Weller, E. Seyed Sadr, E. Willscher, K. Seystahl, G. Reifenberger, G. Tabatabai, H. Binder, H. Schneider, Limited role for transforming growth factor-beta pathway activation-mediated escape from VEGF inhibition in murine glioma models, Neuro Oncol, 18 (2016) 1610-1621.

[24] J. Reinhard, U. Eichhorn, M. Wiessler, B. Kaina, Inactivation of O(6)-methylguanineDNA methyltransferase by glucose-conjugated inhibitors, Int J Cancer, 93 (2001) 373-379. [25] T. Lammers, P. Koczera, S. Fokong, F. Gremse, J. Ehling, M. Vogt, A. Pich, G. Storm, M. van Zandvoort, F. Kiessling, Theranostic USPIO-loaded microbubbles for mediating and monitoring blood-brain barrier permeation, Adv Funct Mater, 25 (2015) 36-43. 
[26] P. Koczera, L. Appold, Y. Shi, M. Liu, A. Dasgupta, V. Pathak, T. Ojha, S. Fokong, Z. Wu, M. van Zandvoort, O. Iranzo, A.J.C. Kuehne, A. Pich, F. Kiessling, T. Lammers, PBCA-based polymeric microbubbles for molecular imaging and drug delivery, $\mathrm{J}$ Control Release, 259 (2017) 128-135.

[27] K. Hynynen, N. McDannold, H. Martin, F.A. Jolesz, N. Vykhodtseva, The threshold for brain damage in rabbits induced by bursts of ultrasound in the presence of an ultrasound contrast agent (Optison), Ultrasound Med Biol, 29 (2003) 473-481.

[28] J. Ramalho, R.C. Semelka, M. Ramalho, R.H. Nunes, M. AlObaidy, M. Castillo, Gadolinium-based contrast agent accumulation and toxicity: an update, AJNR Am J Neuroradiol, 37 (2016) 1192-1198.

[29] Q. Lin, K.-L. Mao, F.-R. Tian, J.-J. Yang, P.-P. Chen, J. Xu, Z.-L. Fan, Y.-P. Zhao, W.F. Li, L. Zheng, Y.-Z. Zhao, C.-T. Lu, Brain tumor-targeted delivery and therapy by focused ultrasound introduced doxorubicin-loaded cationic liposomes, Cancer Chemother Pharmacol, 77 (2016) 269-280.

[30] T. Kato, A. Natsume, H. Toda, H. Iwamizu, T. Sugita, R. Hachisu, R. Watanabe, K. Yuki, K. Motomura, K. Bankiewicz, T. Wakabayashi, Efficient delivery of liposomemediated MGMT-siRNA reinforces the cytotoxity of temozolomide in GBM-initiating cells, Gene Ther, 17 (2010) 1363-1371.

[31] B. Yoo, M.A. Ifediba, S. Ghosh, Z. Medarova, A. Moore, Combination treatment with theranostic nanoparticles for glioblastoma sensitization to TMZ, Mol Imaging Biol, 16 (2014) 680-689.

[32] Y.-N. Zhang, W. Poon, A.J. Tavares, I.D. McGilvray, W.C.W. Chan, Nanoparticle-liver interactions: Cellular uptake and hepatobiliary elimination, J Control Release, 240 (2016) 332-348.

[33] Y. Shen, Z. Pi, F. Yan, C.K. Yeh, X. Zeng, X. Diao, Y. Hu, S. Chen, X. Chen, H. Zheng, Enhanced delivery of paclitaxel liposomes using focused ultrasound with microbubbles for treating nude mice bearing intracranial glioblastoma xenografts, Int J Nanomedicine, 12 (2017) 5613-5629.

[34] H. Schneider, E. Szabo, R.A.C. Machado, A. Broggini-Tenzer, A. Walter, M. Lobell, D. Heldmann, F. Süssmeier, S. Grünewald, M. Weller, Novel TIE-2 inhibitor BAY-826 displays in vivo efficacy in experimental syngeneic murine glioma models, J Neurochem, 140 (2017) 170-182.

[35] M.R. Gilbert, M. Wang, K.D. Aldape, R. Stupp, M.E. Hegi, K.A. Jaeckle, T.S. Armstrong, J.S. Wefel, M. Won, D.T. Blumenthal, A. Mahajan, C.J. Schultz, S. Erridge, B. Baumert, K.I. Hopkins, T. Tzuk-Shina, P.D. Brown, A. Chakravarti, W.J. Curran, Jr., M.P. 
Mehta, Dose-dense temozolomide for newly diagnosed glioblastoma: a randomized phase III clinical trial, J Clin Oncol, 31 (2013) 4085-4091.

[36] C. Happold, P. Roth, W. Wick, N. Schmidt, A.M. Florea, M. Silginer, G. Reifenberger, M. Weller, Distinct molecular mechanisms of acquired resistance to temozolomide in glioblastoma cells, J Neurochem, 122 (2012) 444-455.

[37] A.W. Tolcher, S.L. Gerson, L. Denis, C. Geyer, L.A. Hammond, A. Patnaik, A.D.

Goetz, G. Schwartz, T. Edwards, L. Reyderman, P. Statkevich, D.L. Cutler, E.K. Rowinsky, Marked inactivation of O6-alkylguanine-DNA alkyltransferase activity with protracted temozolomide schedules, Br J Cancer, 88 (2003) 1004-1011.

[38] D.M. Kokkinakis, D.B. Bocangel, S.C. Schold, R.C. Moschel, A.E. Pegg, Thresholds of O6-alkylguanine-DNA alkyltransferase which confer significant resistance of human glial tumor xenografts to treatment with 1,3-Bis(2-chloroethyl)-1-nitrosourea or temozolomide, Clin Cancer Res, 7 (2001) 421-428.

[39] H.S. Friedman, S. Keir, A.E. Pegg, P.J. Houghton, O.M. Colvin, R.C. Moschel, D.D. Bigner, M.E. Dolan, O-6-benzylguanine-mediated enhancement of chemotherapy, Mol Cancer Ther, 1 (2002) 943-948.

[40] M. Clemons, J. Kelly, A.J. Watson, A. Howell, R.S. McElhinney, T.B. McMurry, G.P. Margison, O6-(4-bromothenyl)guanine reverses temozolomide resistance in human breast tumour MCF-7 cells and xenografts, Br J Cancer, 93 (2005) 1152-1156.

[41] M.R. Middleton, N. Thatcher, T.B.H. McMurry, R.S. McElhinney, D.J. Donnelly, G.P. Margison, Effect of O6-(4-bromothenyl)guanine on different temozolomide schedules in a human melanoma xenograft model, Int J Cancer, 100 (2002) 615-617.

[42] J. Hu, J. Wang, G. Wang, Z. Yao, X. Dang, Pharmacokinetics and antitumor efficacy of DSPE-PEG2000 polymeric liposomes loaded with quercetin and temozolomide:

Analysis of their effectiveness in enhancing the chemosensitization of drug-resistant glioma cells, Int J Mol Med, 37 (2016) 690-702.

[43] M. Wu, Y. Fan, S. Lv, B. Xiao, M. Ye, X. Zhu, Vincristine and temozolomide combined chemotherapy for the treatment of glioma: a comparison of solid lipid nanoparticles and nanostructured lipid carriers for dual drugs delivery, Drug Deliv, 23 (2016) 2720-2725. [44] M. Westphal, D.C. Hilt, E. Bortey, P. Delavault, R. Olivares, P.C. Warnke, I.R. Whittle, J. Jaaskelainen, Z. Ram, A phase 3 trial of local chemotherapy with biodegradable carmustine (BCNU) wafers (Gliadel wafers) in patients with primary malignant glioma, Neuro Oncol, 5 (2003) 79-88.

[45] D. Allhenn, M.A.S. Boushehri, A. Lamprecht, Drug delivery strategies for the treatment of malignant gliomas, Int J Pharm, 436 (2012) 299-310. 
1 [46] D.S. Bidros, M.A. Vogelbaum, Novel drug delivery strategies in neuro-oncology,

2 Neurotherapeutics, 6 (2009) 539-546.

3 [47] A. Carpentier, M. Canney, A. Vignot, V. Reina, K. Beccaria, C. Horodyckid, C.

4 Karachi, D. Leclercq, C. Lafon, J.-Y. Chapelon, L. Capelle, P. Cornu, M. Sanson, K.

5 Hoang-Xuan, J.-Y. Delattre, A. Idbaih, Clinical trial of blood-brain barrier disruption by

6 pulsed ultrasound, Sci Transl Med, 8 (2016) 343re342-343re342.

7 [48] A. Idbaih, M. Canney, A. Vignot, B. Law-Ye, C. Dehais, C. Houillier, F. Laigle-

8 Donadey, J.-Y. Delattre, A. Carpentier, Phase I/II study of an implantable device delivering

9 low intensity pulsed ultrasound (LIPU) to disrupt the blood-brain barrier (BBB) followed by

10 intravenous carboplatin chemotherapy in patients with recurrent glioblastoma (GBM), J

11 Clin Oncol, 35 (2017) 2034-2034.

12

13

14

15

16

17

18 


\section{Supplementary Figure 1}

A

3

4 Supplementary Figure 1. Cryo-TEM images of liposomal formulations. Samples of

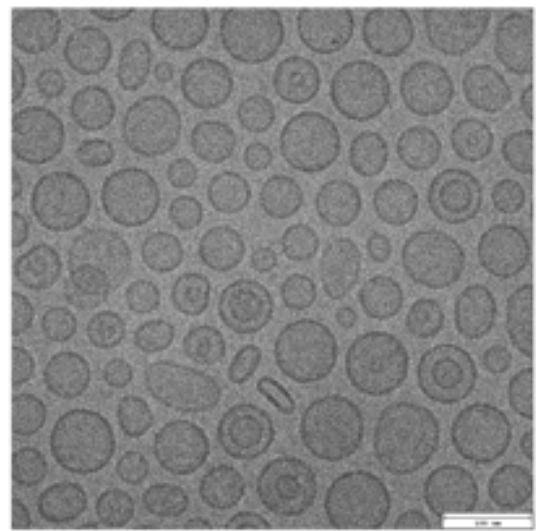

B

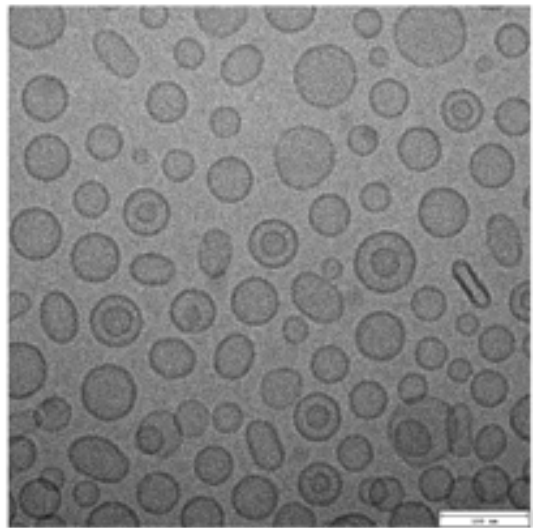

5 control vehicle (A) and LP-O'B BTG-C18 (B) at a drug loading of $4 \mathrm{~mol} \%$ were added to a

6 copper grid covered by holey carbon film, snap-frozen into liquid ethane and transferred

7 into the pre-cooled cryo-electron microscope. Representive images for each formulation

8 are shown. This figure was reproduced from reference [17] with permission.

9 
1 Supplementary Table 1. Hydrodynamic diameter and PDI of empty liposomes and

2 LP-O ${ }^{6}$ BTG-C18 at drug concentrations of $4 \mathrm{~mol} \%$. Results are expressed as means 3 and SD $(n=3)$.

4 5

6

7

8

9

10

11

12

13

14

15

16

17

18

19

20

21

22

23

24

25

26

Hydrodynamic

PDI

diameter $(\mathrm{nm})$

Empty liposomes

$76.0 \pm 0.3$

$0.034 \pm 0.013$

LP-O'BTG-C18

$71.4 \pm 0.5$

$0.052 \pm 0.007$

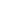

( 1 2 3 4 5 6

7 8 9 0 1 2 3 4 5 6 


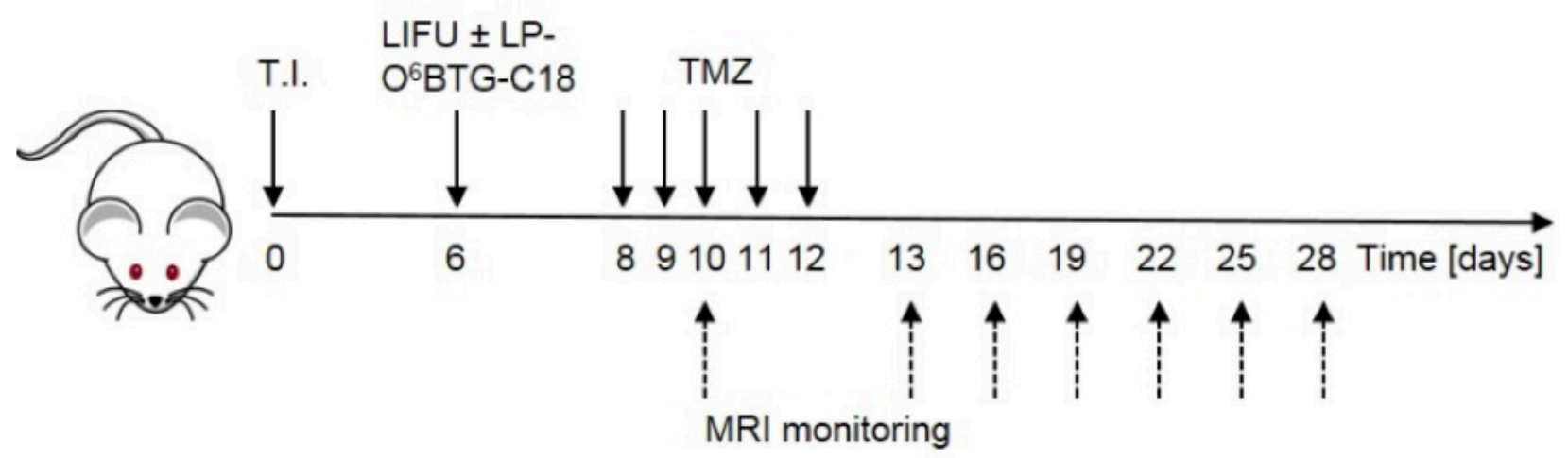

2

3 Supplementary Figure 2. Treatment plan. Five $\times 10^{3}$ SMA-497 glioma cells were

4 injected stereotactically into the right hemisphere of VM/Dk mice (T.I.). Six days after

5 tumor inoculation, mice were intravenously injected with vehicle control or LP-O ${ }^{6} \mathrm{BTG}-\mathrm{C} 18$

$6 \quad(7.2 \mathrm{mg} / \mathrm{kg})$. The LIFU protocol was applied targeting the tumor area via MRI. Two days

7 after tumor LIFU treatment, systemic oral treatment with TMZ (10 mg/kg) or DMSO control

8 was initiated for five consecutive days. T2-weighted MRI tumor volume monitoring was

9 initiated ten days after tumor inoculation and then every three days for three pre-

10 randomized animals. 

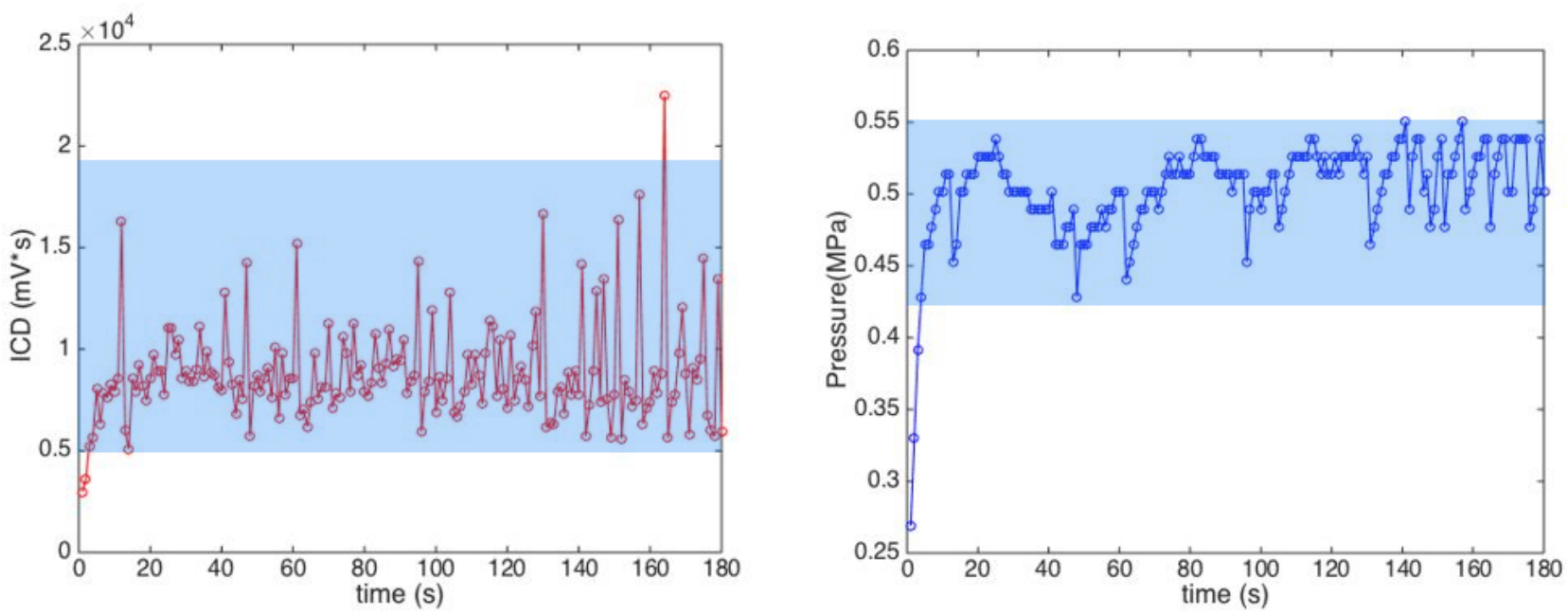

3 Supplementary Figure 3. Microbubble feedback loop for LIFU power control. Mice were intravenously infused with $50 \mu \mathrm{L}$ softshell BG8235 microbubbles at a rate of $1 \mu \mathrm{L} / \mathrm{s}$ for a duration of $50 \mathrm{~s}$ in total. LIFU was applied using a transducer with a PCD and, was

6 delivered in bursts of $10 \mathrm{~ms}$ at a repetition frequency of $1 \mathrm{~Hz}$ for a total duration of $180 \mathrm{~s}$.

$7 \quad$ Very short $(200 \mu \mathrm{s})$ bursts of ultrasound were emitted and the acquired inertial cavitation

8 dose (ICD) signal from the softshell microbubbles was analysed for specific signatures that

9 are indicative for the threshold where desired stable cavitation turns into unwanted inertial cavitation. Applied LIFU power (right panel) was adaptively modulated to keep the instant ICD (left panel) within the limits of the operation band (highlightend blue area) over $180 \mathrm{~s}$. This protocol was applied to each individual mouse for LIFU treatment. Representative ICD and LIFU power data for one mouse are shown. 


\section{Supplementary Figure 4}
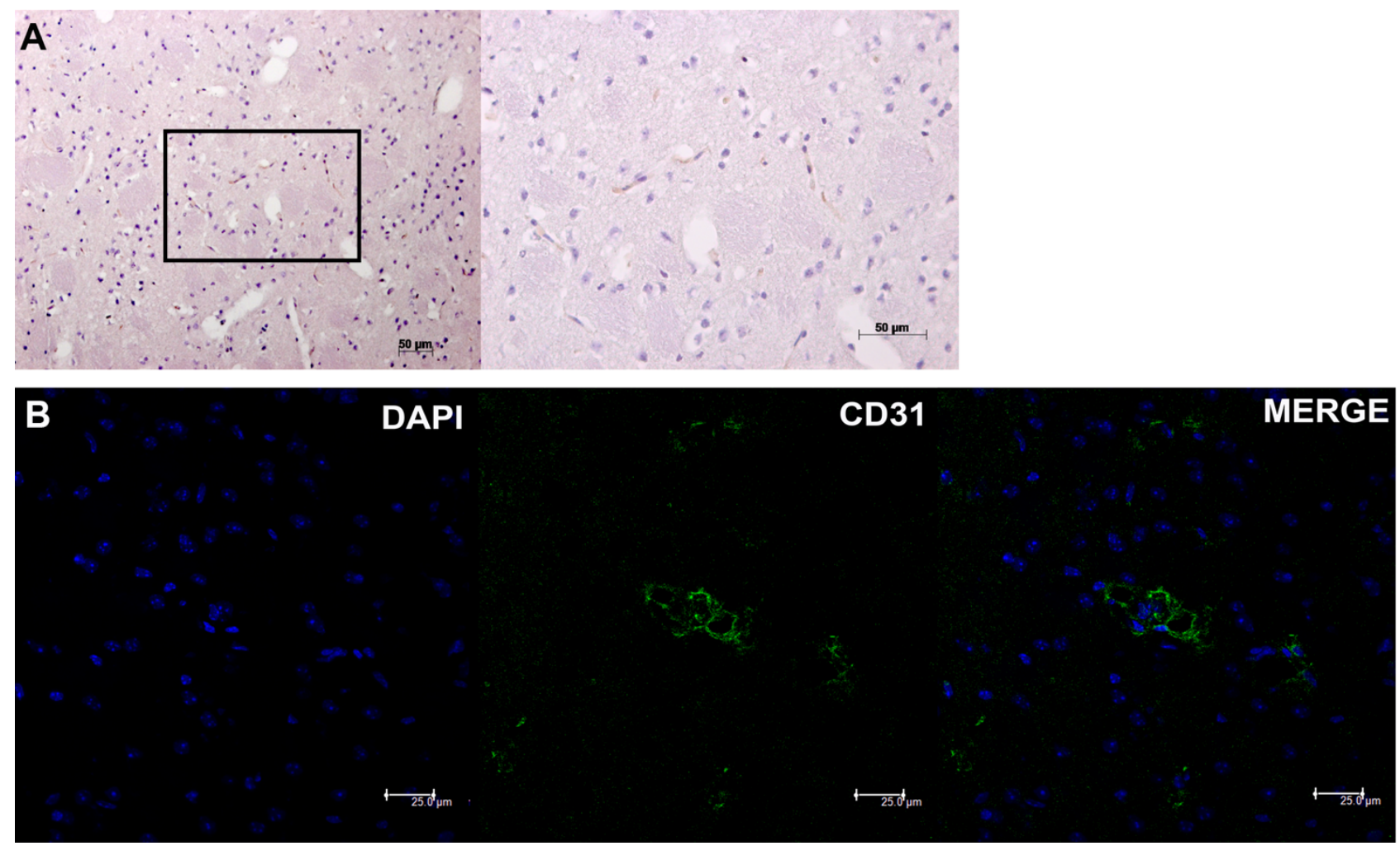

Supplementary Figure 4. Blood vessel staining in brain sections from LIFU-treated

mice. A-B. Healthy mice were intravenously injected with DiD-labelled liposomes (0.3

$4 \mu \mathrm{g} / \mathrm{kg}$ ). LIFU was applied targeting a specific region in the right hemisphere. Hematoxylin

5 and Eosin (H\&E) staining of LIFU-treated brain area (A), black square indicates $40 \mathrm{x}$ area

6 magnification on the right side. CD31staining for blood vessels (green) and nuclear DAPI

7 (blue) staining at 60x magnification (B). Representative images from one mouse $(n=3)$. 
Supplementary Figure 5

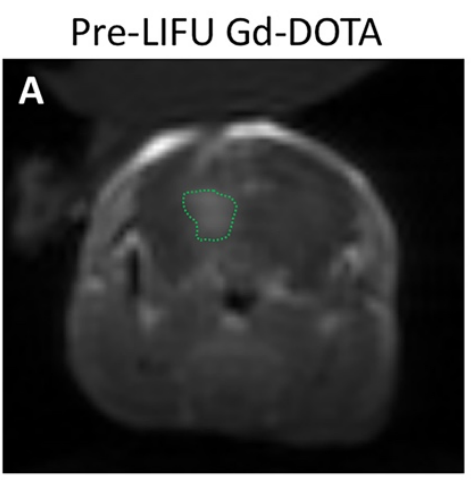

Post-LIFU Gd-DOTA
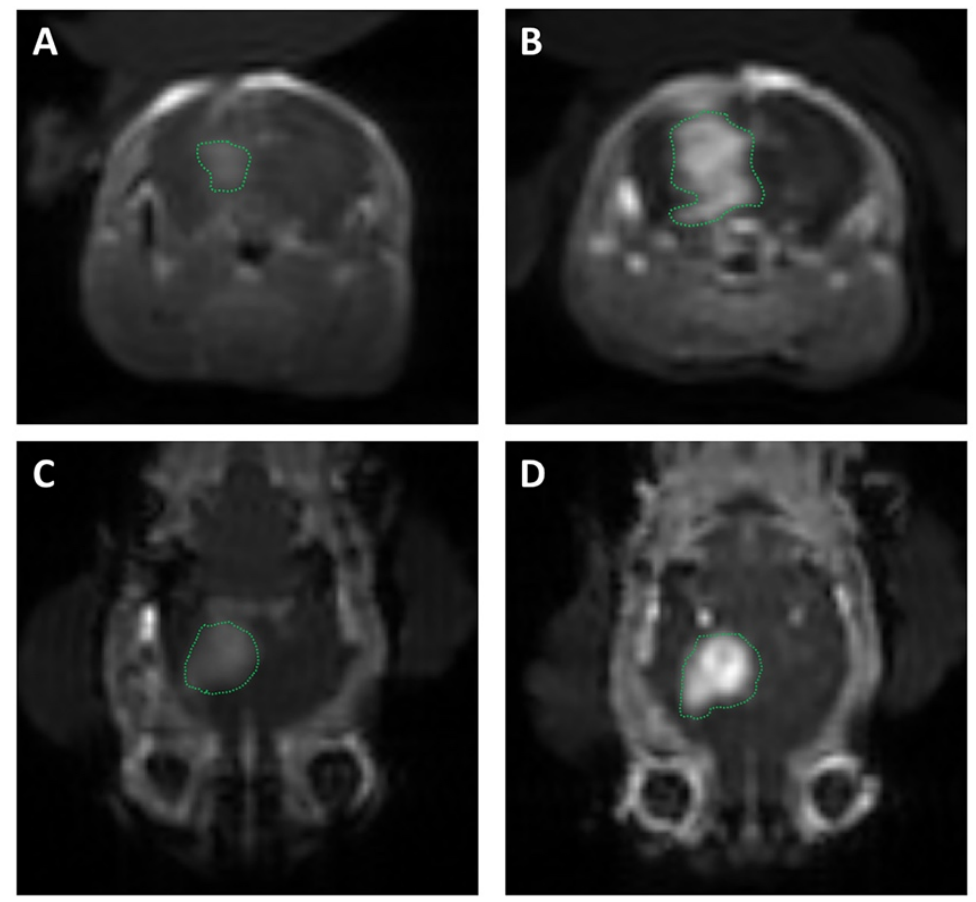

Supplementary Figure 5. Gadolinium (Gd-DOTA) enhancement MRI before and after

4 LIFU-mediated BBB opening. Five $\times 10^{3}$ SMA-497 glioma cells were injected

5 stereotactically into the right hemisphere of VM/Dk mice. Six days after tumor implantation,

6 the LIFU protocol was applied targeting the tumor area via MRI. BBB opening was

7 visualized by T1-weighted Gd-DOTA-enhanced MRI before (A, C) and after LIFU (B, D) in

8 selected mice. Contrast enhancement indicates the extent of Gd-DOTA extravasation as

9 depicted in green dashed lines. Representative sagittal (A, B) and axial (C, D) MR images

10 of one mouse are shown. 

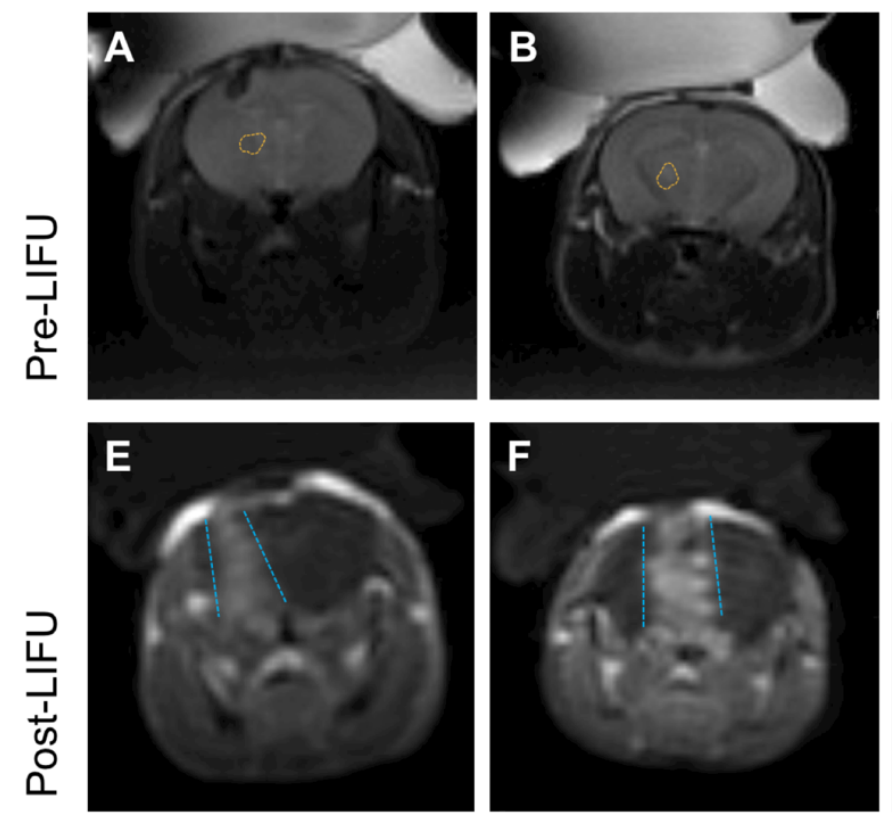

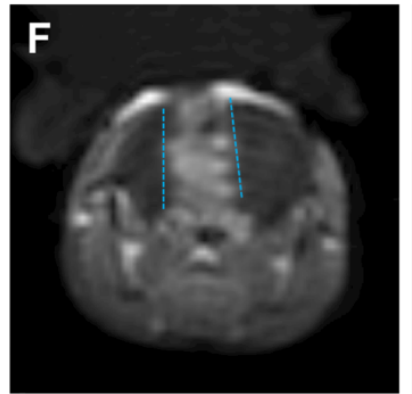

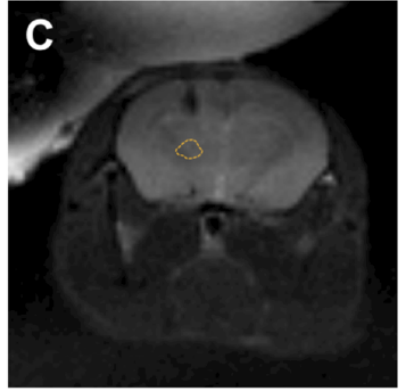

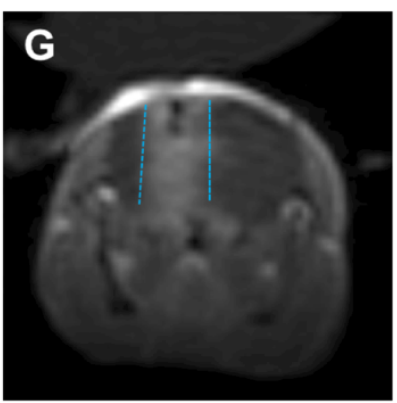

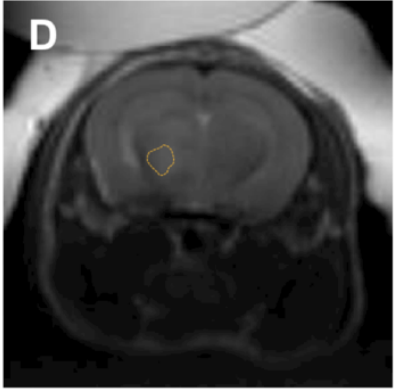

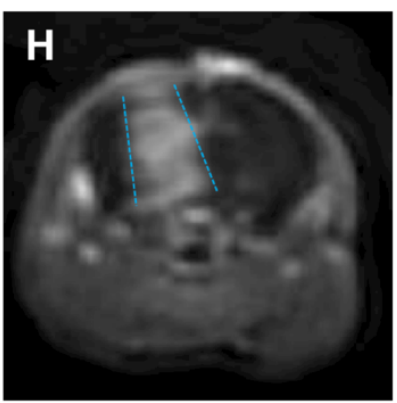

Supplementary Figure 6. LIFU-mediated BBB opening in different treatment groups.

Five $\times 10^{3}$ SMA-497 glioma cells were injected stereotactically into the right hemisphere of

VM/Dk mice. Six days after tumor implantation, the LIFU protocol was applied targeting the tumor area (depicted in yellow dashed lines) via MRI alone (A, E), with TMZ (B, F) or LP$\mathrm{O}^{6} \mathrm{BTG}-\mathrm{C} 18(\mathbf{C}, \mathbf{G})$ or with TMZ plus LP-O'BTG-C18 (D, H). BBB opening was visualized by T1-weighted Gd-DOTA-enhanced MRI. Contrast enhancement indicates the extent of Gd-DOTA extravasation within the path of targeted LIFU sonication as depicted in blue dashed lines $(E-H)$. Representative images of one mouse per group are shown. 


\section{Supplementary Figure 7}

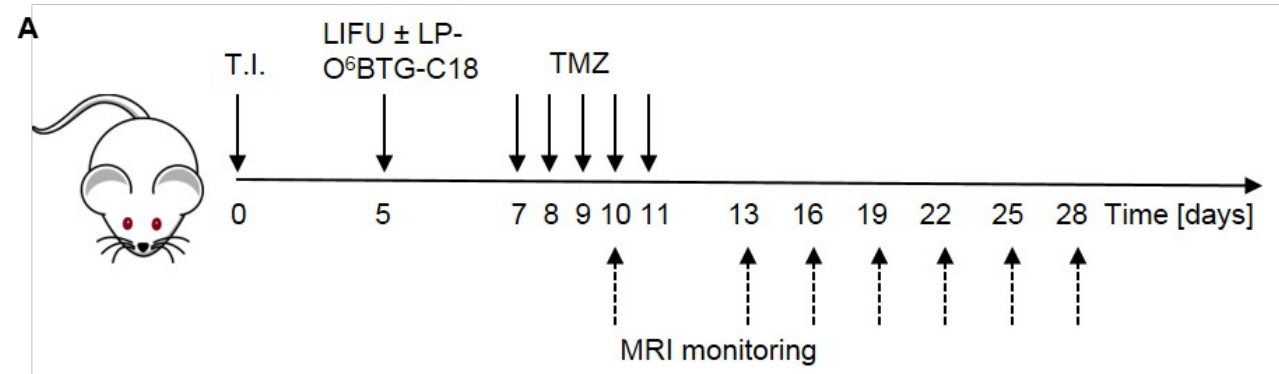

B
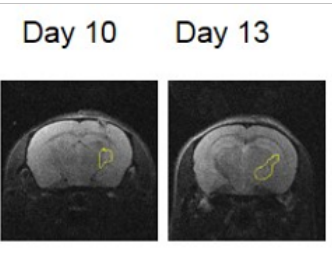

Day 16

Day 19

Day 22

Day 25

Day 28

LIFU

TMZ
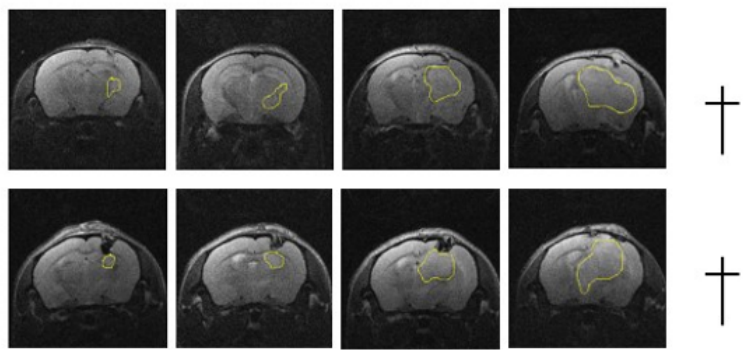

TMZ+LPO'BTG-

C18
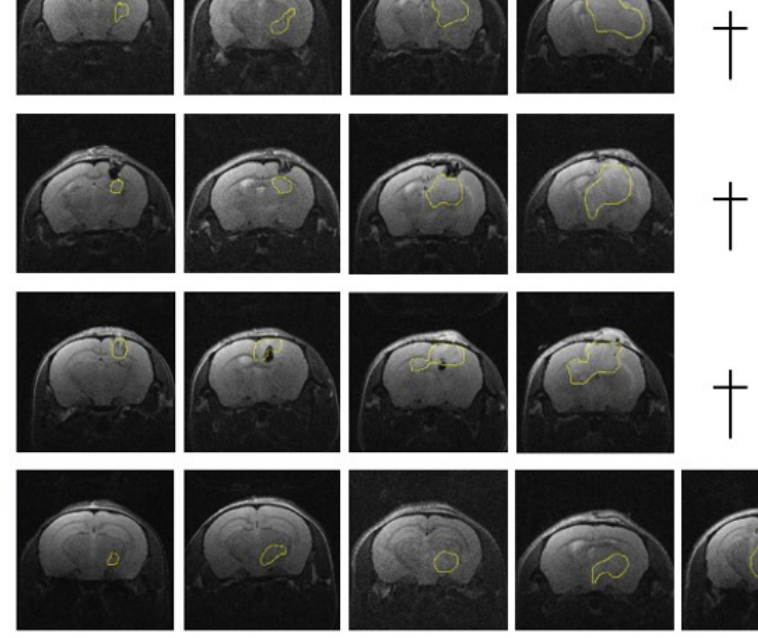

LIFU+TMZ
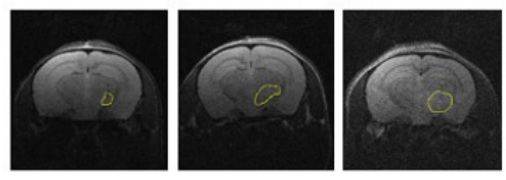

LIFU+ TMZ+LPO6BTGC18
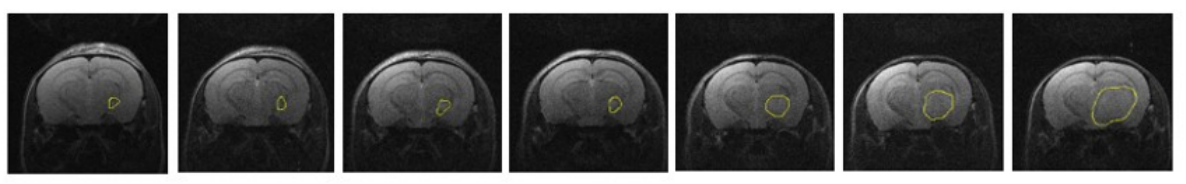

C

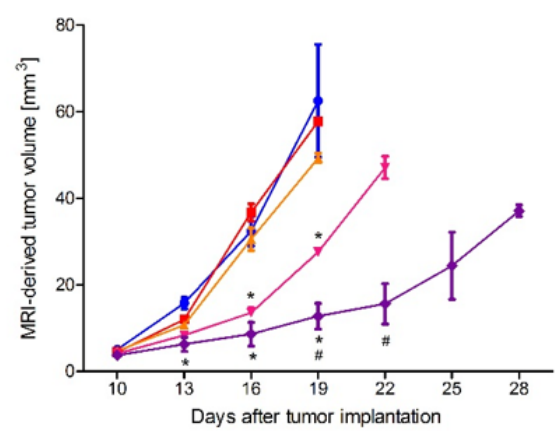

D

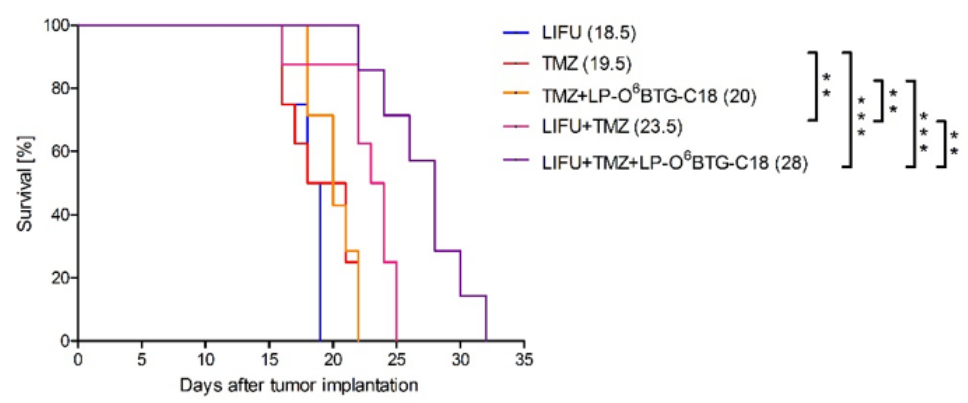

3 Supplementary Figure 7. Systemic LP-O ${ }^{6}$ BTG-C18 in combination with LIFU and TMZ

4 treatment reduces tumor growth and prolongs survival in glioma-bearing mice. A.

5 Treatment plan: Five $\times 10^{3}$ SMA-497 glioma cells were injected stereotactically into the right

6 hemisphere of VM/Dk mice (T.I.). Five days after tumor inoculation, mice were intravenously 
1 injected with vehicle control or LP-O6 BTG-C18 $(7.2 \mathrm{mg} / \mathrm{kg})$. The FUS protocol was applied

2 targeting the tumor area via MRI. Two days after tumor LIFU treatment, systemic oral

3 treatment with TMZ (10 mg/kg) or DMSO control was initiated for five consecutive days. B,

4 C. T2-weighted MRI tumor volume monitoring was initiated ten days after tumor inoculation

5 and then every three days for three pre-randomized animals. Representative MRI images

6 of one mouse per group are shown with the yellow lines depicting the tumor area (B).

7 Crosses $(\dagger)$ indicate that the animals had to be sacrificed due to score 2 neurological

8 symptoms. Tumor volume measurements are expressed as means and SD $(n=3)$, statistical

9 analysis was performed with one-way ANOVA and Bonferroni post-hoc testing with * or \# $p$ $10<0.05 .{ }^{*}=$ TMZ alone vs LIFU+TMZ or LIFU+TMZ+LP-O ${ }^{6}$ BTG-C18; \# = LIFU+TMZ vs

11 LIFU+TMZ+LP-O'B BTG-C18 (C). D. Mice were treated as in (A) and sacrificed when they 12 developed grade 2 neurological symptoms. Kaplan-Meier survival curves are shown with 13 median survival depicted in parentheses for each group. Survival curves were analyzed for 14 differences via Log-rank (Martel-Cox) test with ${ }^{*} p<0.05$ or ${ }^{* * *} p<0.001$. 
1 Supplementary Table 2. Survival range. Survival curves were analyzed for

2 differences via Log-rank (Martel-Cox) test with $p$ values as indicated.

\begin{tabular}{|c|c|c|c|c|c|c|c|c|c|}
\hline $\begin{array}{l}\text { Treatment } \\
\text { group }\end{array}$ & $\begin{array}{l}\text { Mean } \\
\text { survival } \\
\text { [days] }\end{array}$ & $\begin{array}{l}\text { Median } \\
\text { survival } \\
\text { [days] }\end{array}$ & $\begin{array}{l}p- \\
\text { value }^{a}\end{array}$ & $\begin{array}{l}p- \\
\text { value }^{b}\end{array}$ & $\begin{array}{l}p- \\
\text { value }^{c}\end{array}$ & $\begin{array}{l}p- \\
\text { value }^{d}\end{array}$ & $\begin{array}{l}p- \\
\text { value }^{\mathrm{e}}\end{array}$ & $\begin{array}{l}p- \\
\text { value }^{f}\end{array}$ & $\begin{array}{l}p- \\
\text { value }^{g}\end{array}$ \\
\hline Untreated $(n=4)$ & 15 & 13 & - & - & - & - & - & - & - \\
\hline $\begin{array}{l}\text { LP-O }{ }^{6} \text { BTG-C18 } \\
(n=4)\end{array}$ & 15 & 13.5 & 0.265 & - & - & - & - & - & - \\
\hline $\begin{array}{l}\text { LIFU+LP- } \\
\text { OBTG-C18 } \\
(n=7)\end{array}$ & 16 & 14 & 0.239 & 0.246 & - & - & - & - & - \\
\hline LIFU $(n=4)$ & 14 & 13.5 & 0.193 & 0.371 & 0.370 & - & - & - & - \\
\hline $\operatorname{TMZ}(\mathrm{n}=4)$ & 16 & 14.5 & 0.096 & 0.108 & 0.204 & 0.158 & - & - & - \\
\hline $\begin{array}{l}\text { TMZ+LP- } \\
\text { O }^{6} \text { BTG-C18 } \\
(n=4)\end{array}$ & 16 & 14 & 0.060 & 0.064 & 0.0102 & 0.390 & 0.531 & - & - \\
\hline $\begin{array}{l}\text { LIFU+TMZ } \\
(n=7)\end{array}$ & 19 & 17.5 & 0.011 & 0.015 & 0.0103 & 0.010 & 0.037 & 0.018 & - \\
\hline $\begin{array}{l}\text { LIFU+TMZ+LP- } \\
\text { O'BTG-C18 }^{6} \\
(n=7)\end{array}$ & 30 & 27 & $<0.001$ & $<0.001$ & $<0.001$ & $<0.001$ & $<0.001$ & $<0.001$ & $<0.001$ \\
\hline
\end{tabular}

${ }^{a}$ compared to untreated; ${ }^{b}$ compared to LP-O ${ }^{6}$ BTG-C18; ${ }^{c}$ compared to LIFU+LP-O 6 BTG-C18;

4

${ }^{d}$ Compared to LIFU; ${ }^{e}$ compared to TMZ; ${ }^{\text {f }}$ compared to TMZ+LP-O ${ }^{6}$ BTG-C18; ${ }^{9}$ Compared to LIFU+TMZ 

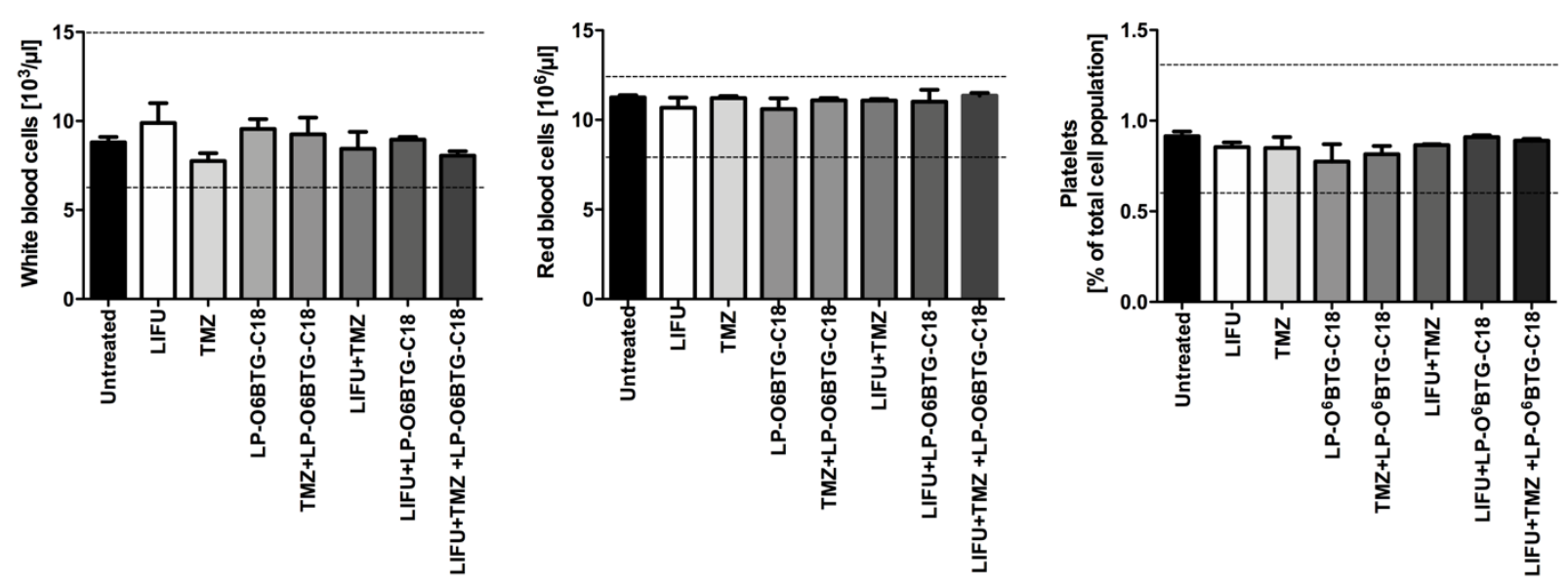

3 Supplementary Figure 8. LIFU in combination with systemic LP-O6 BTG-C18 and TMZ

4 treatment does not increase hematological toxicity. A-C. Five $\times 10^{3}$ SMA-497 glioma

5 cells were injected stereotactically into the right hemisphere of VM/Dk mice. Five days

6 after tumor inoculation, the mice were intravenously injected with control vehicle or LP-

7 O ${ }^{6} \mathrm{BTG}-\mathrm{C} 18(7.2 \mathrm{mg} / \mathrm{kg})$. The LIFU protocol was applied targeting the tumor area via MRI.

8 Two days after tumor LIFU treatment, systemic oral treatment with TMZ (10 mg/kg) or

9 DMSO control was initiated for five consecutive days. Three days after completion of TMZ

10 treatment, blood samples were collected from three pre-randomized animals from each

11 group. White blood cell $(A)$, red blood cell $(B)$ and platelet $(C)$ populations were measured

12 as a percentage of the total cell population. Dotted lines depict the normal range of each

13 analyzed condition as provided by the laboratory for veterinary medicine (University of

14 Zurich, Zurich, Switzerland). Results are expressed as means and SD ( $n=3)$, statistical

15 analysis was performed with one-way ANOVA and Bonferroni post-hoc testing with * $p$

$16<0.05$. 


\section{Graphical Abstract}

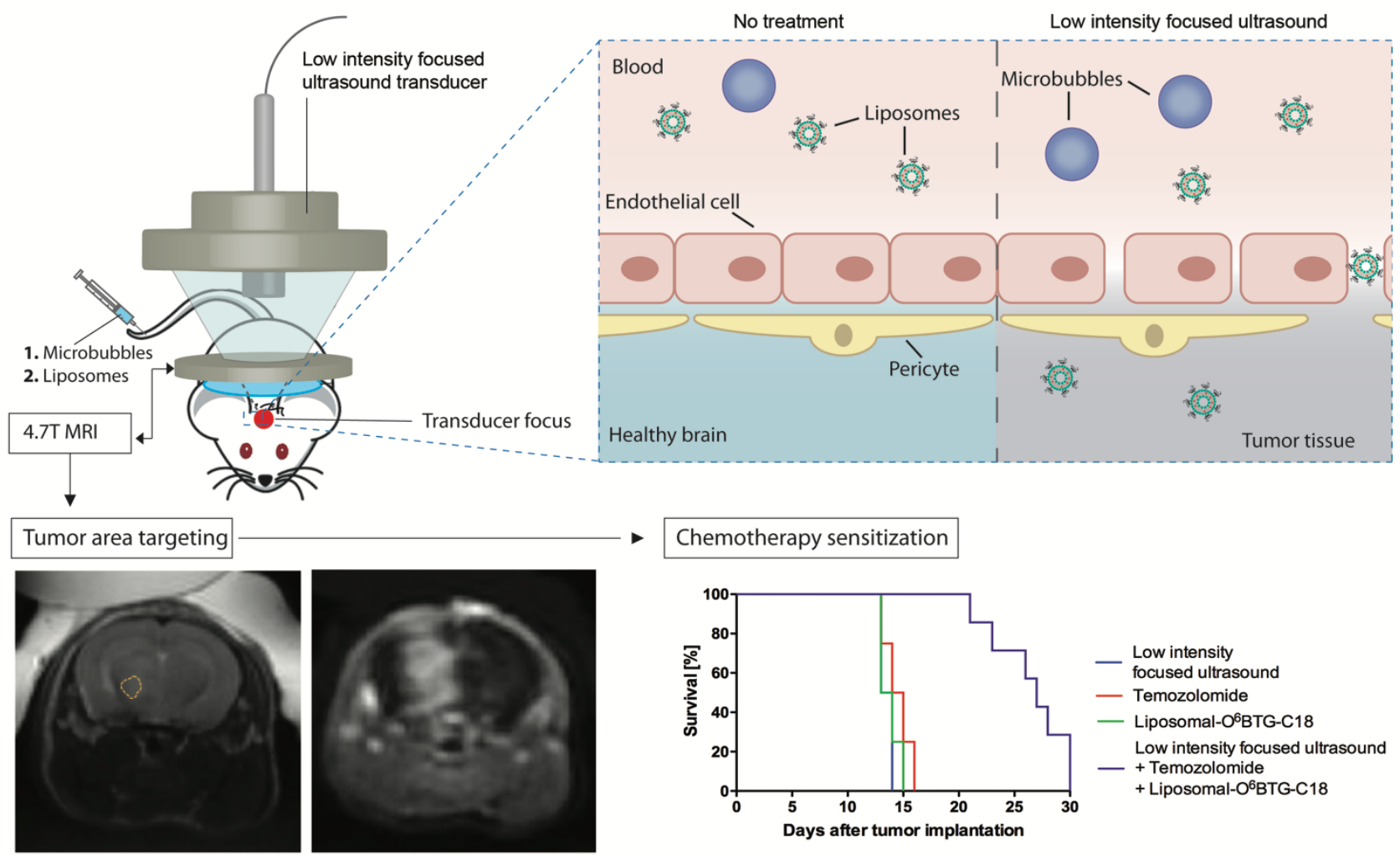

